\title{
Die „Würdigung aller Umstände des Einzelfalls“ - ein unbekanntes Phänomen
}

Thomas Riehm*

A. Einleitung ......................... 1

B. Einzelfallabwägung und

Justizsyllogismus ....................

I. Abwägungsentscheidungen im Justizsyllogismus .................. 4

II. Die Subsumtion als eigenständiger Schritt der Rechtsanwendung........ 5

III. Der abstrakt-generelle Charakter des Obersatzes ........................ 7

IV. Geschlossene und wertungsoffene Obersätze ........................ 10

V. Ergebnis: Einzelfallabwägung als Subsumtion ..................... 11

C. Die Struktur der Einzelfallabwägung . . 11

I. Die Auswahl des Abwägungsmaterials ............... 12

II. Die Gewichtung der Abwägungsaspekte................ 14

1. Abstrakte Gewichtung.......... 14

2. Konkrete Gewichtung........... 15

III. Die Ermittlung des Ergebnisses...... 16

IV. Die Wertungslast................... 17

1. Möglichkeit eines non liquet.... 18

2. Funktionen der Wertungslast.... 19

3. Regelungen der Wertungslast... 20
a) Die Vermutung des Vorlie- gens eines Handelsgewerbes ( $\$ 1$ Abs. 2 HGB) .
1

4

4

5

7

0

1

D. Kon

D. Kontrollmaßstäbe fï......

Einzelfallabwägungen................ 26

I. Gegenstand der Kontrolle ............ 26

II. Prozedurale Abwägungsregeln....... 29

III. Verbleibende nicht falsifizierbare

Elemente der Abwägung........... 31

E. Rechtstheoretische Folgerungen...... 32

I. Einzelfallabwägung und Rationalität rechtlicher Entscheidungen........ 32

II. Einzelfallabwägung und „One Right Answer"-These ....................... 34

III. Einzelfallabwägung und Topik...... 36

F. Zusammenfassung ................. 38

\section{A. Einleitung}

„Diese Frage ist vom Tatrichter unter Würdigung aller Umstände des Einzelfalls zu entscheiden “ - unzählige Urteile des BGH in Zivilsachen, aber auch in Strafsachen und Revisionsurteile anderer Gerichtszweige verweisen auf eine solche „Würdigung“, „Abwägung“ oder „Berücksichtigung aller Umstände des Einzelfalls“. ${ }^{1}$ Bei näherer Betrachtung dient diese Formulierung zwei verschiedenen Zwecken: Zum einen betrifft sie die Tatsachenfeststellung und verweist dabei auf das Erfordernis

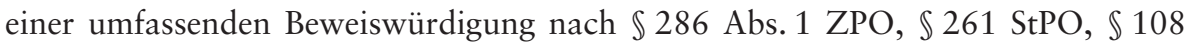

* Prof. Dr. Thomas Riehm ist Inhaber des Lehrstuhls für Bürgerliches Recht, Rechtsvergleichung, Europäisches Privatrecht und Zivilverfahrensrecht an der Philipps-Universität Marburg.

1 Die Datenbank juris weist aus allen Gerichtszweigen insgesamt 6.847 Treffer für „Würdigung aller Umstände“, 3.693 für „Abwägung aller Umstände“ und 11.656 für „Berücksichtigung aller Umstände“ nach, zusammen also mehr als 22.000 Treffer (Stand: 12.12.2012). Da auch die Rechtsprechung nicht zwischen den drei Begriffen „Abwägung “, „Würdigung“ und „Berücksichtigung “ unterscheidet, werden sie im Folgenden synonym verwendet. 
Abs. 1 VwGO u. ä. ${ }^{2}$ Diese Fälle sind in der beweisrechtlichen Literatur umfassend aufgearbeitet ${ }^{3}$ und nicht Gegenstand des folgenden Beitrags.

Diese Abhandlung befasst sich vielmehr mit dem zweiten Anwendungsfall, der Einzelfallwürdigung im Zusammenhang mit der Anwendung unbestimmter bzw. wertausfüllungsbedürftiger Tatbestandsmerkmale, etwa bei der Konkretisierung des „groben Missverhältnisses“ zwischen Leistungsaufwand und Schuldnerinteresse in $\$ 275$ Abs. 2 BGB, ${ }^{4}$ bei der Bestimmung der ,groben Fahrlässigkeit“ ${ }^{5}$ oder des Vorliegens eines „wichtigen Grundes“ für die Abberufung eines WEG-Verwalters, ${ }^{6}$ bei der Auslegung von Willenserklärungen nach $\mathbb{S} \mathbb{1 3 3}, 157 \mathrm{BGB}^{7}$ oder bei der Bestimmung der Leistungskürzung eines Versicherers wegen einer Obliegenheitsverletzung des Versicherungsnehmers, ${ }^{8}$ um nur einige wenige Beispiele aus jüngerer Zeit zu zitieren. Eine Würdigung aller Umstände des Einzelfalles findet aber auch bei verwaltungsrechtlichen Ermessensentscheidungen ${ }^{9}$ oder Ausübungen von Beurteilungsermächtigungen statt, ${ }^{10}$ und nicht zuletzt bei der Strafzumessung (vgl. $\$ 46$ Abs. 2 S. 1 StGB) ${ }^{11}$

So allgegenwärtig diese Form der „Abwägung aller Umstände des Einzelfalls“ in der Rechtspraxis ist, so überraschend ist es, dass ihre Struktur und ihr Inhalt bisher kaum näher untersucht worden sind. ${ }^{12}$ Das gilt sowohl für ihre rechtstheoretische Einordnung in den Prozess der Rechtsanwendung als auch für ihre Behandlung im Verfahrensrecht der unterschiedlichen Gerichtszweige. ${ }^{13}$ In rechtstheoretischer Hinsicht drängt sich die Frage nach der Art der Entscheidung auf, die dem Tatgericht durch

2 Vgl. etwa BGH WM 2012, 954, 958 (Rn. 30) zur Feststellung bedingten Vorsatzes; BGH NStZ 2012, 160, 161 (Rn. 25) zur Feststellung eines vorsatzausschließenden Tatbestandsirrtums.

3 Vgl. für das Zivilprozessrecht etwa G. Baumgärtel/H.-W. Laumen/H. Prütting (Hrsg.), Handbuch der Beweislast, Bd. 1: Grundlagen, 2. Aufl., Köln u. a. 2009, für den Strafprozess z. B. G. Freund, Normative Probleme der „Tatsachenfeststellung“, Heidelberg 1987.

4 BGH NJW 2010, S. 2050 (2052, Rn. 23).

5 BGH NJW 2008, S. 3123 (3124, Rn. 15).

6 BGH NJW 2012, S. 1884 (1885, Rn. 10).

7 BGH NJW-RR 2012, S. 741 (742, Rn. 8).

8 BGH NJW-RR 2012, S. 724 (725, Rn. 13).

9 Vgl. nur BVerfGE 35, 382 (401); 50, 166 (176); BVerwGE 15, 196 (202); 56, 56 (59); H.-J. Koch/H. Rüßmann, Juristische Begründungslehre, München 1982, S. 236 ff.; H.-J. Koch, Die normtheoretische Basis der Abwägung, in: W. Erbguth/J. Oebbecke u. a. (Hrsg.), Abwägung im Recht, Köln u. a. 1996, S. 9 (22 f.); R. Bartlsperger, Das Abwägungsgebot in der Verwaltung als objektives und individualrechtliches Erfordernis konkreter Verhältnismäßigkeit, ebd., S. 79 (103); M. Borowski, Intendiertes Ermessen, DVBl. 2000, S. 149 (151); näher T. Riehm, Abwägungsentscheidungen in der praktischen Rechtsanwendung, München 2006, S. 46 ff.

10 Vgl. dazu etwa H.-J. Papier, Verwaltungsverantwortung und gerichtliche Kontrolle, in: W. Blümel (Hrsg.), FS Ule, Köln u. a. 1987, S. 235 (242); Riehm, Abwägungsentscheidungen (Fn. 9), S. 51 f.

11 Vgl. hierzu - mit durchaus kritischer Tendenz, aber nicht bezogen auf die Strafzumessung selbst - E. Horn, Gesamtwürdigung - Sinn und Unsinn eines Rechtsbegriffs, in: G. Dornseifer (Hrsg.), GS Armin Kaufmann, Köln u. a. 1989, S. 573 ff.

12 Vgl. immerhin Horn, Gesamtwürdigung (Fn. 11).

13 S. dazu Riehm, Abwägungsentscheidungen (Fn. 9), S. 233 ff. 
diese Formulierung abverlangt wird. Schon der erste Anschein spricht dafür, dass es sich um eine Abwägungsentscheidung handelt, bei welcher diverse Umstände des Sachverhalts ermittelt, gesammelt, gewichtet und gegeneinander abgewogen werden sollen. Teilt man vorläufig diesen Ausgangspunkt, so fragt sich allerdings, welche Rolle eine solche im Justizsyllogismus spielen kann, der doch (vermeintlich) auf eine schlichte Subsumtion eines Sachverhalts unter eine Rechtsnorm, also nach herkömmlichem Verständnis gerade nicht auf eine Abwägungsentscheidung ausgerichtet ist. ${ }^{14}$ Häufig wird sogar ein strenger Gegensatz zwischen „normenstrenger“ Subsumtion und „freier“ Abwägung heraufbeschworen, ${ }^{15} \mathrm{zu}$ dem eine solche Verweisung auf die tatrichterliche Abwägung innerhalb der richterlichen Rechtsanwendung gar nicht passen will. ${ }^{16}$

Der Verweis auf die Abwägung aller Umstände des Einzelfalls zielt offensichtlich darauf ab, dem Tatgericht eine Entscheidung zu ermöglichen, die nicht streng regelgeleitet ist, sondern ihm einen gewissen Entscheidungsspielraum belässt. Dass es sich gleichwohl nicht um eine willkürliche Entscheidungsmacht handelt, ist schon daraus ersichtlich, dass die Revisionsgerichte sich gleichwohl eine - wenn auch eingeschränkte - Kontrolle der tatrichterlichen Würdigung vorbehalten; ${ }^{17}$ eine Befugnis der Instanzgerichte zu willkürlichen Entscheidungen wäre ohnehin verfassungsrechtlich schon nach Art. 3 Abs. 1 GG ausgeschlossen. Vielmehr existieren auch für die Einzelfallwürdigung Regeln, die wenn schon nicht das Abwägungsergebnis, so doch den Abwägungsvorgang vorgeben und jedenfalls eine prozedurale Falsifikation der so getroffenen Entscheidungen ermöglichen.

Der folgende Beitrag versucht, ausgehend von einer Analyse der Struktur derartiger Abwägungsentscheidungen die Möglichkeit ihrer Richtigkeitskontrolle bzw. Falsifikation zu untersuchen, um schließlich zu ihrer Rationalität und Berechtigung in einem rechtsstaatlichen Rechtssystem Stellung zu nehmen.

14 Vgl. zur üblichen Gegenüberstellung von Abwägungs- und Subsumtionsmodell nur R. Alexy, Die Abwägung in der Rechtsanwendung, in: Meiji Gakuin Universität (Hrsg.), Jahresbericht des Institutes für Rechtswissenschaften an der Meiji Gakuin Universität, Tokio 2001, S. 69 ff.; ders., On Balancing and Subsumption, Ratio Juris 16 (2003), S. $433 \mathrm{ff.}$

15 Vgl. etwa - jeweils bezogen auf die verfassungsrechtliche Güterabwägung - W. Leisner, „Abwägung überall“ - Gefahr für den Rechtsstaat, NJW 1997, S. 636 ff.; ders., Der Abwägungsstaat, Berlin 1997; K.-H. Ladeur, Kritik der Abwägung in der Grundrechtsdogmatik, Tübingen 2004; J. Rückert, Abwägung - die juristische Karriere eines unjuristischen Begriffs oder: Normenstrenge und Abwägung im Funktionswandel, JZ 2011, S. 913 ff.

$16 \mathrm{Zu} \mathrm{Recht} \mathrm{kritisch} \mathrm{in} \mathrm{Bezug} \mathrm{auf} \mathrm{unstrukturierte} \mathrm{Einzelfallabwägungen} \mathrm{auch} \mathrm{G.} \mathrm{Freund,} \mathrm{Über} \mathrm{die} \mathrm{Be-}$ deutung der „Rechts“-Folgenlegitimation für eine allgemeine Theorie juristischer Argumentation, JZ 1992, S. 993 (997).

17 Vgl. BGHZ 10, 14 (18); 20, 290 (292 f.); 51, 275 (279f.); 55, 45 (55); NJW 2004, 3413 (3415); E. Schwinge, Grundlagen des Revisionsrechts, 2. Aufl., Bonn 1960, S. 113 ff.; K. Kuchinke, Grenzen der Nachprüfbarkeit tatrichterlicher Würdigung und Feststellungen in der Revisionsinstanz, Bielefeld 1964, S. 132 ff., insbes. S. 142 f.; W. Grunsky, in: Stein/Jonas (Hrsg.), Zivilprozessordnung, 21. Aufl., Tübingen 1993, $\$ \$ S 549,550$ Rn. 21 ff., 28 ff.; L. Rosenberg/K. H. Schwab/P. Gottwald, Zivilprozessrecht, 17. Aufl., München 2010, \$141 Rn. 31 ff.; eingehend Riehm, Abwägungsentscheidungen (Fn. 9), S. 186 ff. 


\section{B. Einzelfallabwägung und Justizsyllogismus}

Die Entscheidungsstruktur der Abwägung ist zunächst aus dem Verfassungsrecht bekannt, namentlich aus der Abwägung kollidierender Grundrechte - diese schlägt auch auf das Zivilrecht durch. ${ }^{18}$ Sie ist aber auf den ersten Blick schwer in Einklang zu bringen mit dem herkömmlichen Subsumtionsmodell, wonach sich die richterliche Aufgabe bei der Rechtsanwendung auf die Subsumtion eines Sachverhalts unter einen Obersatz beschränkt, der seinerseits aus einer Rechtsnorm gewonnen wurde. ${ }^{19}$ Gleichwohl besteht kein Zweifel daran, dass in den einschlägigen Fällen im Grundsatz eine Subsumtion unter eine Rechtsnorm stattfindet. Die Einzelfallabwägung ist lediglich in diese integriert, bildet also einen Teil davon.

\section{Abwägungsentscheidungen im Justizsyllogismus}

Wie bereits an anderer Stelle ausführlich dargelegt wurde, ${ }^{20}$ ist dies nichts Ungewöhnliches: Bei näherer Betrachtung finden auf allen Stufen des Justizsyllogismus Abwägungsentscheidungen statt: Bei der Obersatzbildung werden Auslegungsargumente zusammengetragen, zunächst nach ihrer Art (z. B. Wortlaut, Systematik, Entstehungsgeschichte, Normzweck) ${ }^{21}$ und dann nach ihrer konkreten Überzeugungskraft gewichtet, und schließlich gegeneinander abgewogen, um aus einem abstrakt formulierten Gesetzestext einen subsumtionsfähigen, auf den Sachverhalt zugeschnittenen Obersatz zu gewinnen. ${ }^{22}$ Bei der Feststellung des Sachverhalts werden ebenfalls Beweismittel gesammelt, nach ihrer Art (z. B. Urkunden, Zeugen, Sachverständige $)^{23}$ und ihrer konkreten Überzeugungskraft gewichtet, und gegeneinander abgewogen, um zu ermitteln, ob eine bestimmte Tatsachenbehauptung wahr oder unwahr ist (vgl. auch $\mathbb{2} 286 \mathrm{ZPO}$ ). Ziel ist in beiden Fällen eine richterliche Überzeugung: Im einen Fall von der Richtigkeit der gefundenen Gesetzesauslegung in Ge-

18 Vgl. BGHZ 131, 332 ff.; 183, 227 ff.; BGH NJW 2008, 3134; s. dazu auch H. Hubmann, Wertung und Abwägung im Recht, Köln u. a. 1977, S. 145 ff.; C.-W. Canaris, Das Recht auf Meinungsfreiheit gemäß Art. 5 Abs. 1 GG als Grundlage eines arbeitsrechtlichen Kontrahierungszwangs, in: J. Isensee/ H. Lecheler (Hrsg.), FS Leisner, Berlin 1999, S. 5 ff.; ders., Grundrechte und Privatrecht, Berlin 1999, S. $62 \mathrm{ff}$.

19 Vgl. die Nachweise oben Fn. 14.

20 Vgl. Riehm, Abwägungsentscheidungen (Fn. 9), S. 4 ff.

21 Vgl. zur Rangfolge der Auslegungskriterien nur C.-W. Canaris, Das Rangverhältnis der „klassischen“ Auslegungskriterien, demonstriert an Standardproblemen aus dem Zivilrecht, in: V. Beuthien (Hrsg.), FS Medicus, Köln u. a. 1999, S. 25 ff.

22 Vgl. dazu etwa K. Engisch, Logische Studien zur Gesetzesanwendung, 3. Aufl., Heidelberg 1963, S. 16 f.; H.-E. Henke, Rechtsfrage oder Tatfrage - eine Frage ohne Antwort?, ZZP 81 (1968), S. 196 (219).

23 Vgl. zu möglichen Gewichtungsregeln für Beweismittel G. Freund, Die Tatfrage als Rechtsfrage, in: A. Eser (Hrsg.), FS Meyer-Goßner, München 2001, S. 409 (425 ff.). 
stalt eines subsumtionsfähigen Obersatzes, im anderen Fall von der Wahrheit oder Unwahrheit einer Tatsachenbehauptung. ${ }^{24}$

Betrachtet man diese beiden Formen der Abwägung, so lässt sich die „Würdigung aller Umstände des Einzelfalls“ allerdings keiner der beiden Abwägungen zuordnen: Von der Obersatzbildung unterscheidet sie sich dadurch, dass ihr Ziel nicht die Festlegung einer (zwar gegenüber dem Gesetzestext konkretisierten, aber immer noch) abstrakt-generellen Regel ist, sondern die auf den Einzelfall bezogene individuellkonkrete Aussage, ob ein bestimmtes wertausfüllungsbedürftiges Tatbestandsmerkmal gegeben ist oder nicht. Der Unterschied zur Beweiswürdigung besteht darin, dass ihr Ziel nicht die Feststellung eines empirisch messbaren Sachverhalts ist, sondern die normative Bewertung eines hinsichtlich seiner Fakten bereits festgestellten Sachverhalts: Ob besondere Umstände „unter Abwägung der beiderseitigen Interessen die sofortige Geltendmachung des Schadensersatzanspruchs“ bzw. „den sofortigen Rücktritt rechtfertigen“ (vgl. $\mathbb{S} \$ 281$ Abs. 2 Alt. 2, 323 Abs. 2 Nr. 3 BGB), ist keine empirische, einer Beweiserhebung zugängliche Frage, sondern eine Bewertungsfrage, die das Gericht erst auf der Basis der beweismäßig festgestellten Tatsachen beantworten kann. Dementsprechend ist die Einzelfallwürdigung auch nicht empirisch auf ihre Richtigkeit überprüfbar, sondern nur normativ, also durch eine erneute Bewertung derselben Umstände durch eine andere Instanz. ${ }^{25}$ Die Einzelfallwürdigung kann daher nicht - wie die Beweiswürdigung - empirisch „wahr“ oder „unwahr“ sein, sondern nur normativ „richtig“ (bzw. „,vertretbar“) oder „falsch“.26

\section{Die Subsumtion als eigenständiger Schritt der Rechtsanwendung}

Gehört die Einzelfallabwägung danach weder zur Obersatzbildung noch zur Tatsachenfeststellung, so bleibt unter Zugrundelegung des herkömmlichen Justizsyllogismus nur die Möglichkeit, sie der Subsumtion zuzuordnen. Üblicherweise ist allerdings der Schritt der Subsumtion ein äußerst trivialer, der häufig gar nicht als besonderer Gedankenschritt wahrgenommen wird: Hat man einerseits auf der Ebene des Obersatzes ermittelt, dass der Schuldner einer unmöglich gewordenen Leistung

24 Vgl. dazu und zu weiteren Parallelen eingehend Riehm, Abwägungsentscheidungen (Fn. 9), S. 57 ff.; s. auch U. Neumann, Subsumtion als regelorientierte Fallentscheidung, in: G. Gabriel/R. Gröschner (Hrsg.), Subsumtion, Tübingen 2012, S. 311 (312 ff.).

25 Diese rechtstheoretische Einordnung sagt allerdings noch nichts darüber aus, ob es sich im revisionsrechtlichen Sinne um eine Tatfrage oder um eine Rechtsfrage handelt (vgl. dazu Riehm, Abwägungsentscheidungen [Fn. 9], S. 233 ff. m. w. N.).

26 Vgl. zu dieser elementaren erkenntnistheoretischen Unterscheidung etwa Freund, „Rechts“-Folgenlegitimation (Fn. 16), S. 994 m. w. N.; J. Bung, Der juristische Syllogismus in der Methodenlehre von Larenz, in: Gabriel/Gröschner (Hrsg.), Subsumtion (Fn. 24), S. 215 (218 f., 224). Von der Strömung des „moral realism“ wird diese Unterscheidung zwar immer wieder angegriffen (vgl. etwa R. N. Boyd, How to Be a Moral Realist, in: G. Sayre-McCord (Hrsg.), Essays on moral realism, Ithaca, N. Y 1988, S. $181 \mathrm{ff}$.); im vorliegenden Zusammenhang ist das allerdings ohne Bedeutung, solange nicht auch behauptet wird, dass die „objektive Wahrheit“ eines Werturteils tatsächlich mit empirischen Methoden objektiv ermittelbar ist. 
von seiner Naturalleistungspflicht frei wird ( $\$ 275$ Abs. 1 BGB), und andererseits auf der Ebene des Untersatzes (also der Beweiswürdigung) festgestellt, dass die Leistung des A naturgesetzlich unmöglich ist (z. B. weil das verkaufte Kunstwerk bei einem Brand vernichtet wurde), so erscheint der Subsumtionsschluss, dass A von seiner Naturalleistungspflicht frei ist, ebenso zwingend wie trivial. ${ }^{27}$

Indessen ist darin ein wesentlicher Schritt versteckt, der angesichts der Trivialität der Aufgabe leicht außer Acht gerät: Vor dem eigentlichen logischen Schluss, der eine abstrakt-generelle Regel (, der Schuldner einer unmöglich gewordenen Leistung wird von der Naturalleistungspflicht befreit") in eine konkret-individuelle Aussage verwandelt („A wird von seiner Naturalleistungspflicht befreit“), steht die Zuordnung des Lebenssachverhalts „das von A geschuldete Kunstwerk ist verbrannt“ zum Tatbestandsmerkmal „die Leistung ist unmöglich geworden““. ${ }^{28}$ Sprachlogisch wird hier eine konkrete lebensweltliche Tatsache, die empirisch festgestellt wurde, einem Rechtsbegriff zugeordnet. ${ }^{29}$ Diese Zuordnung ist in der Tat im Regelfall trivial, nämlich dann, wenn der Obersatz nur noch aus deskriptiven Tatbestandsmerkmalen besteht, die ihre unmittelbare Entsprechung in der empirischen Lebenswelt haben. ${ }^{30}$ Denn dann können lebensweltliches Ergebnis der Sachverhaltsfeststellung und deskriptiver Rechtsbegriff im Obersatz ohne weiteres zur Deckung gebracht werden, ohne dass dieser Akt überhaupt separat ins Bewusstsein tritt.

Anders liegt es aber, wenn der Obersatz noch wertungsoffene (normative) Tatbestandsmerkmale wie z. B. „angemessen“, „billigerweise“, „zumutbar“, „erforderlich " o. ä. enthält: Hier kann der festgestellte lebensweltliche Sachverhalt nicht ohne weiteres mit dem Rechtsbegriff zur Deckung gebracht werden, weil noch eine richterliche Bewertung der Fakten erforderlich ist, um festzustellen, ob etwa ein bestimmtes (empirisch feststellbares) Zahlenverhältnis zwischen Leistungsaufwand des Gläubigers und Leistungsinteresse des Schuldners ein „grobes Missverhältnis“ i. S. v. $\$ 275$ Abs. 2 BGB begründet oder nicht. Die Subsumtion setzt in diesem Fall ein Werturteil voraus, das nicht seinerseits ein Syllogismus oder mit Hilfe eines Syllogismus' zu gewinnen ist, ${ }^{31}$ sondern ein Weg, innerhalb des Syllogismus' den Schritt der Subsumtion zu bewältigen, also eine besondere Subsumtionsoperation, die sich

27 Vgl. auch R. Gröschner, Justizsyllogismus? Jurisprudenz!, in: K. D. Lerch (Hrsg.), Recht verhandeln, Berlin, New York 2005, S. 203 (204): „Mehr als ein Kinderspiel der Logik ist der Justizsyllogismus demnach nicht.".

28 Vgl. K. Larenz/C.-W. Canaris, Methodenlehre der Rechtswissenschaft, 3. Aufl., Berlin u. a. 1995, S. 37 ff.; T. van Zantwijk, Subsumtion in aristotelischer Tradition, in: Gabriel/Gröschner (Hrsg.), Subsumtion (Fn. 24), S. 25 (35 ff.).

29 Vgl. dazu aus sprachanalytischer Sicht U. Neumann, Recht als Struktur und Argumentation, BadenBaden 2008, S. $14 \mathrm{ff}$.

30 Vgl. Larenz/Canaris, Methodenlehre (Fn. 28), S. 36.

31 So zutreffend H. Arendt/R. Beiner, Das Urteilen, München 1998, S. 14; Gröschner, Justizsyllogismus? Jurisprudenz! (Fn. 27), S. 204 f. 
nicht in einem Vergleich von lebensweltlichem Sachverhalt und deskriptivem Tatbestandsmerkmal erschöpft.

\section{Der abstrakt-generelle Charakter des Obersatzes}

Die Existenz wertausfüllungsbedürftiger Tatbestandsmerkmale im Obersatz setzt allerdings voraus, dass es derartige wertungsoffene Obersätze überhaupt gibt, dass also die letztlich angewendeten Obersätze nicht stets so weit konkretisiert sein müssen, dass sie nur noch deskriptive Tatbestandsmerkmale enthalten, die im Wege einer einfachen Zuordnung subsumiert werden können. Ein wertungsoffener Obersatz wäre dann eben noch nicht vollständig konkretisiert, so dass vor der Subsumtion noch eine weitere Konkretisierung auf Regelebene erforderlich wäre, bis keine wertausfüllungsbedürftigen Tatbestandsmerkmale mehr enthalten sind. Dies entspricht etwa der Lehre von der Fallnorm, ${ }^{32}$ die davon ausgeht, dass der letztlich angewandte Obersatz so weit konkretisiert ist, dass er genau auf den zu lösenden Fall passt - und auf keinen anderen mehr ${ }^{33}$-, und nur noch deskriptive Tatbestandsmerkmale enthält. In eine ähnliche Richtung gehen Ansätze, die den Rechtsanwendungsprozess bei wertausfüllungsbedürftigen Tatbestandsmerkmalen nicht mehr in die drei Stufen Obersatz, Untersatz, Subsumtion einteilen, sondern als kontinuierlichen Konkretisierungsprozess von der abstrakt-generellen Rechtsnorm bis zur konkret-individuellen Einzelfallentscheidung ansehen wollen. ${ }^{34}$

Nach diesen Auffassungen beschränkt sich die Subsumtion in der Tat in jedem Fall auf den bloßen Abgleich von lebensweltlicher Sachverhaltsbeschreibung und deskriptivem Tatbestandsmerkmal; für eine Abwägung ist hier kein Raum mehr. Indessen würde ein derart abschließend bis zur Fallnorm konkretisierter Obersatz die Zwecke verfehlen, denen der Obersatz dient: ${ }^{35}$ Ist der Obersatz so weit konkretisiert,

32 Vgl. dazu v. a. W. Fikentscher, Methoden des Rechts, Tübingen 1975-1977, Bd. IV, S. 202 ff. (382); ebenso die Lehre von der „Entscheidungsnorm“ bei F. Müller/R. Christensen, Juristische Methodik, 10. Aufl., Berlin 2009, S. 204 ff.; ähnlich W. A. Scheuerle, Beiträge zur Trennung von Tat- und Rechtsfrage, AcP 157 (1958/59), S. 1 (38 ff., 49 f., 76); Kuchinke, Grenzen der Nachprüfbarkeit (Fn. 17), S. 77; J. Esser, Vorverständnis und Methodenwahl in der Rechtsfindung, Frankfurt a. M. 1972, S. 56 ff.; B. Schünemann, Zum Verhältnis von Norm und Sachverhalt bei der Rechtsanwendung, von Ober- und Untersatz im Justizsyllogismus und von Rechts- und Tatfrage im Prozessrecht, in: F. Haft (Hrsg.), FS Arthur Kaufmann, Heidelberg 1993, S. 299 (313).

33 Insoweit a. A. (und wie hier) Neumann, Subsumtion (Fn. 24), S. 327: Die Fallnorm beziehe sich ",auf alle Lebenssachverhalte, bei denen dieselben Relevanzmerkmale identifiziert werden.".

34 Vgl. etwa W. Hassemer, Tatbestand und Typus, Köln 1968, S. 99 ff.; J. Rödig, Die Theorie des gerichtlichen Erkenntnisverfahrens, Berlin u.a. 1973, S. 165 ff.; Schünemann, Verhältnis (Fn. 32), S. 313; Gröschner, Justizsyllogismus? Jurisprudenz! (Fn. 27), S. 212 f.; ähnlich auch H. G. Gadamer, Hermeneutik I, 7. Aufl., Tübingen 2010, S. 336: „Konkretisierung des Gesetzes im jeweiligen Fall“; A. Kaufmann, Das Verfahren der Rechtsgewinnung, München 1999, S. 79 u. ö.; s. allgemein zur Rechtsanwendung als Konkretisierungsprozess Kuchinke, Grenzen der Nachprüfbarkeit (Fn. 17), S. 115; K. Engisch, Die Idee der Konkretisierung in Recht und Rechtswissenschaft unserer Zeit, 2. Aufl., Heidelberg 1968, S. 120 ff.; F. Bydlinski, Juristische Methodenlehre und Rechtsbegriff, 2. Aufl., Wien/ New York 1991, S.11; C. Seiler, Auslegung als Normkonkretisierung, Heidelberg 2000, passim; A. Röthel, Normkonkretisierung im Privatrecht, Tübingen 2004, passim.

35 Vgl. zum Folgenden eingehend Riehm, Abwägungsentscheidungen (Fn. 9), S. 19 ff., 26 ff. 
dass er nur noch auf den einen zu entscheidenden Fall passt, so handelt es sich gewissermaßen um einen „Einweg-Obersatz“, der auf der Tatbestandsseite sämtliche (in ihrer Kombination unwiederholbaren) Umstände des konkreten Einzelfalles enthält und dafür eine Rechtsfolge festlegt. Er mag zwar formal noch abstrakt-generell formuliert sein, hat aber im materiellen Sinne keine Bedeutung über den konkreten Einzelfall hinaus, weil die abstrakt-generell formulierten Voraussetzungen einzig und allein in diesem Fall erfüllt sein können, der in der Vergangenheit geschehen ist.

Diese Bedeutung über den konkreten Einzelfall hinaus ist aber ein sowohl aus rechtstheoretischen und wissenschaftstheoretischen als auch aus rechtspraktischen Gründen unabdingbares Charakteristikum des Obersatzes. In rechtstheoretischer Hinsicht sichert der abstrakt-generelle Charakter die mit der Rechtsidee unverzichtbar verbundene systematische Ordnungsfunktion des Rechts: ${ }^{36}$ Erst die Abstraktion von einem konkreten Sachverhalt, die generalisierende Benennung der jeweils wesentlichen Sachverhaltselemente als Tatbestandsmerkmale, erlaubt es überhaupt, von rechtlichen Regeln zu sprechen, an welchen die Rechtsunterworfenen ihr eigenes Verhalten für die Zukunft ausrichten können. ${ }^{37}$ Das gilt auch für die Rechtsprechung selbst: Ohne Abstraktion der angewandten Entscheidungsregel (also des Obersatzes) vom konkreten Sachverhalt bliebe sie bloße Kadijustiz und könnte weder Rechtssicherheit noch Gleichheit in der Rechtsanwendung gewährleisten. ${ }^{38}$

Auch in wissenschaftstheoretischer Hinsicht ist die Abstraktheit des Obersatzes von entscheidender Bedeutung: Der wissenschaftliche Diskurs kann nur über „allgemeine" Sätze stattfinden, ${ }^{39}$ nicht über konkrete Einzelfälle, die von der Rechtsprechung bereits abschließend (rechtskräftig) entschieden sind. Mag sich ein wissenschaftlicher Diskurs auch an einer konkreten Einzelfallentscheidung entzünden - sein Gegenstand kann sinnvollerweise nur die Frage sein, wie vergleichbar gelagerte Fälle in der Zukunft zu entscheiden sein sollen. Dies setzt aber einen abstrakt-generellen Obersatz voraus, der als Entscheidungsregel zukünftigen Entscheidungen zu Grunde gelegt werden kann - über eine „Fallnorm“, welche die Gesamtheit aller unwiederhol-

36 Vgl. dazu C.-W. Canaris, Systemdenken und Systembegriff in der Jurisprudenz, 2. Aufl., Berlin 1983, S. 16, 83, 149 ff.; K. F. Röhl/H. C. Röhl, Allgemeine Rechtslehre, 3. Aufl., München 2008, $\mathbb{S} 78$ IV (S. $621 \mathrm{f}$.$) .$

37 Vgl. zu dieser Funktion juristischer Theorien C.-W. Canaris, Funktion, Struktur und Falsifikation juristischer Theorien, JZ 1993, S. 377 f.; Larenz/Canaris, Methodenlehre (Fn. 28), S. 71; Neumann, Subsumtion (Fn. 24), S. 317 ff.

38 Vgl. zu dieser grundlegenden Funktion des Rechts C.-W. Canaris, Die Feststellung von Lücken im Gesetz, 2. Aufl., Berlin 1983, S. 57; s. ferner Freund, „Rechts“-Folgenlegitimation (Fn. 16), S. 996; K. Langenbucher, Die Entwicklung und Auslegung von Richterrecht, München 1996, S. 41 ff.; U. Neumann, Das Problem der Identität der Rechtsfrage beim Ausgleich divergierender obergerichtlicher Entscheidungen ( $\mathbb{S} 121$ II, 132 II GVG), in: Eser (Hrsg.), FS Meyer-Goßner, S. 683 (691 ff.).

39 Vgl. Canaris, Funktion, Struktur und Falsifikation juristischer Theorien (Fn. 37), S. 377 f. unter Hinweis auf I. Kant, Über den Gemeinspruch: Das mag in der Theorie richtig sein, taugt aber nicht für die Praxis, Berlinische Monatsschrift 1793, S. 201 und K. R. Popper, Logik der Forschung, 10. Aufl., Tübingen 1994, S. 31. 
baren Elemente eines konkreten Lebenssachverhaltes als Tatbestand enthält, kann nicht sinnvoll diskutiert werden.

Schließlich verlangen rechtspraktische Gründe einen abstrakt-generellen Charakter des Obersatzes: Die praktische Bedeutung des Obersatzes einer höchstrichterlichen (Einzelfall-)Entscheidung zeigt sich in seiner Leitbildfunktion für zukünftige Entscheidungen desselben Gerichts oder untergeordneter Gerichte. Besonders deutlich wird das bei den amtlichen Leitsätzen höchstrichterlicher Entscheidungen, die für nachgeordnete Instanzen in der Praxis geradezu die Funktion echter Rechtssätze übernehmen. Im deutschen Prozessrecht wird dem durch die Zulassungsvoraussetzungen für Rechtsmittel Rechnung getragen: Nach $\$ 511$ Abs. 4 S. 1 Nrn. 1, 2 ZPO bzw. $\int 543$ Abs. 2 S. 1 Nrn. 1, 2 ZPO sind Berufung bzw. Revision wegen grundsätzlicher Bedeutung oder zur Sicherung einer einheitlichen Rechtsprechung nur zuzulassen, wenn der vom Untergericht zu Grunde gelegte abstrakte Rechtssatz von einem abstrakten Rechtssatz eines anderen Gerichts abweicht. ${ }^{40}$ Gleiches gilt im Verwaltungsprozess für die Zulassung von Berufung und Revision nach $\$ 124$ Abs. 2 Nrn. 3, 4 VwGO bzw. $\$ 132$ Abs. 2 Nrn. 1, 2 VwGO. Eine Rechtskontrolle kommt also nach der positiv-verfahrensrechtlichen Ausgestaltung nur für abstrakte Rechtssätze in Betracht, nicht für konkrete Einzelfallentscheidungen. Diese gesetzliche Ausgestaltung ist auch sachlich gerechtfertigt, weil der obergerichtlichen Rechtsprechung nur bei abstrakten Rechtssätzen eine Beispielswirkung für künftige Verfahren zukommen kann. ${ }^{41}$ Auch dafür ist also die Formulierung eines abstrakt-generellen Obersatzes erforderlich, der für mehr als nur eine Fallgestaltung tatsächlich Anwendung finden kann. ${ }^{42}$

Wie weit die Konkretisierung des Obersatzes am Ende erfolgen kann, lässt sich nicht generell im Vorhinein sagen. Der Konkretisierungsprozess, der im wissenschaftlichen Diskurs zwischen Rechtsprechung und Literatur stattfindet, führt selbst bei Generalklauseln wie $\mathbb{S} 138$ BGB zu einer stetigen Ausdifferenzierung der Obersätze durch die Bildung von Fallgruppen, Ausnahme-Fallgruppen und Unterausnahmen dazu. Diese bleiben allesamt noch abstrakt-generell formuliert und sind daher auch im hier vertretenen Sinne echte Obersätze, die auf mehrere Fallgestaltungen anwendbar sind.

40 Vgl. nur BGH NJW 2003, 754 (755); 2004, 1960 (1961); BGHZ 151, 42; BGHZ 151, 221 (224); BGHZ 152, 182 (186); B. Rimmelspacher, in: Münchener Kommentar zur Zivilprozessordnung, 4. Aufl., München 2012, $\$ 511$ Rn. 74; W. Ball, in: Musielak (Hrsg.), ZPO, 9. Aufl., München 2012, \$5 $543 \mathrm{Rn} .4 \mathrm{ff}$.

41 Vgl. nur Kuchinke, Grenzen der Nachprüfbarkeit (Fn. 17), S. 141 f.; H.-E. Henke, Die Tatfrage - der unbestimmte Begriff im Zivilrecht und seine Revisibilität, Berlin 1965, S. 9 f., 268.

42 Vgl. etwa BGH NJW-RR 2008, 1565 (Rn. 7) zu $\$ 574$ Abs. 2 S. 1 Nr. 2 Alt. 2 ZPO: „Diese Fragestellung dürfte auch einer näheren abstrakt-generellen Beantwortung kaum zugänglich sein. Sie betrifft eine weitgehend vom Tatrichter anhand der Einzelfallumstände vorzunehmende Abklärung nach den vorgenannten in Rechtsprechung und Rechtslehre nicht umstrittenen Voraussetzungen für ein Geschäftslokal.“. 


\section{Geschlossene und wertungsoffene Obersätze}

Soll der Obersatz aber auf mehrere Fallgestaltungen anwendbar sein, so kann er nicht sämtliche Umstände des Einzelfalles in seinen Tatbestand aufnehmen, sondern muss vom konkreten Sachverhalt abstrahieren, also nicht einen Fall, sondern einen realen Falltypus kennzeichnen. ${ }^{43}$ Damit ist es möglich, dass nicht alle normativen (wertausfüllungsbedürftigen) Tatbestandsmerkmale der Rechtsnorm zu deskriptiven Merkmalen konkretisiert werden. Denn manche gesetzliche Merkmale sind so sehr einzelfallbezogen, dass jeder noch so konkrete abstrakt-generelle Rechtssatz dem anwendenden Gericht noch einen Abwägungsspielraum belassen muss, um etwa atypische Fallgestaltungen und ex ante noch unbekannte Aspekte berücksichtigen zu können. ${ }^{4}$

Folglich können zwei Arten von Obersätzen unterschieden werden: Geschlossene Obersätze, die nur noch deskriptive - d. h. empirisch feststellbare - Tatbestandsmerkmale enthalten, und wertungsoffene Obersätze, die trotz ihrer Konkretisierung noch wertausfüllungsbedürftige Tatbestandsmerkmale enthalten. Dieser Charakter der Obersätze ist nicht zwingend von den in der Rechtsnorm verwendeten Tatbestandsmerkmalen abhängig: Normative Tatbestandsmerkmale in einer Rechtsnorm können durchaus so weit konkretisiert werden, dass der letztlich angewendete Obersatz nur noch deskriptive Tatbestandsmerkmale enthält; ein besonders augenfälliges Beispiel hierfür bildet das Recht des Kindesunterhalts, wo das gesetzliche normative Tatbestandsmerkmal des „angemessenen Unterhalts“ ( $\$ 1601$ BGB) durch die Rechtsprechung der Oberlandesgerichte so weit konkretisiert wurde, dass der Unterhaltsbetrag in Standardfällen aus der "Düsseldorfer Tabelle“ abgelesen werden kann. ${ }^{45}$ Auch bei anderen normativen Tatbestandsmerkmalen gelingt es der Rechtsprechung immer wieder, präzise Fallgruppen herauszubilden, die durch deskriptive Tatbestandsmerkmale abschließend beschrieben werden können.

Zumeist aber verbleibt im Obersatz noch (mindestens) ein Tatbestandsmerkmal, das trotz aller Konkretisierungsbemühungen nicht weiter ausdifferenziert werden kann. Das gilt nicht nur für explizite Abwägungsnormen wie etwa die $\mathbb{S} \$ 281$ Abs. 2 Alt. 2, 286 Abs. 2 Nr. 4 und $\$ 323$ Abs. 2 Nr. 3 BGB, welche explizit eine „Abwägung der beiderseitigen Interessen" verlangen, sondern auch für sonstige normative Tatbestandsmerkmale, die sich allenfalls in Regelbeispielen konkretisieren lassen, etwa

43 Ähnlich Neumann, Identität der Rechtsfrage (Fn. 38), S. 695 f.; ders., Subsumtion (Fn. 24), S. 330 f.: „Hyperkomplexität des Einzelfalls“.

44 Vgl. dazu Röthel, Normkonkretisierung (Fn. 34), S. 184 ff., 211 ff. m. zahlr. Bsp.; s. als Beispiel aus dem Strafrecht BGHSt 28, 165 (167) und dazu Neumann, Identität der Rechtsfrage (Fn. 38), S. 699 ff.

45 Vgl. Röthel, Normkonkretisierung (Fn. 34), S. 171 ff., 282 ff. 
die Sittenwidrigkeit in $\$ 138$ BGB. ${ }^{46}$ Bei derartigen Regelbeispielen tritt die Rechtsfolge nur „in der Regel“ ein, wenn bestimmte (deskriptive) Tatbestandsvoraussetzungen gegeben sind. ${ }^{47}$ Die Regel lässt aber Ausnahmen zu (sog. defeasibility). ${ }^{48}$ Für die Anwendung eines solchen Regelbeispiels muss der Rechtsanwender nach Subsumtion der deskriptiven Tatbestandsmerkmale eine zusätzliche Einzelfallabwägung vornehmen. In deren Rahmen hat er zu prüfen, ob nicht besondere Umstände des Einzelfalles ein Abweichen von der regelmäßigen Rechtsfolge gebieten. Auch hierbei handelt es sich daher letztlich um wertungsoffene Obersätze.

\section{Ergebnis: Einzelfallabwägung als Subsumtion}

Existieren mithin wertungsoffene Obersätze, so können auch im Obersatz noch wertausfüllungsbedürftige Tatbestandsmerkmale verbleiben. Im Hinblick auf diese Tatbestandsmerkmale erfordert die Subsumtion mehr als den bloßen Abgleich eines festgestellten empirischen Sachverhaltselements mit einem deskriptiven Tatbestandsmerkmal: Eben eine Abwägungsentscheidung, die Abwägung (bzw. gleichbedeutend: die Würdigung) aller Umstände des Einzelfalles, um zu beurteilen, ob das wertausfüllungsbedürftige Tatbestandsmerkmal erfüllt ist oder nicht. Diese Abwägungsentscheidung ist demnach weder Obersatzbildung noch Sachverhaltsfeststellung, sondern Subsumtion eines festgestellten Sachverhalts unter einen konkretisierten Obersatz.

\section{Die Struktur der Einzelfallabwägung}

Die Grundstruktur der Einzelfallabwägung ist die gleiche wie bei allen Abwägungsentscheidungen: ${ }^{49}$ Zunächst ist das Abwägungsmaterial auszuwählen, sind also die abwägungsrelevanten Gesichtspunkte zu ermitteln und zu benennen, die für und gegen die Verwirklichung des normativen Tatbestandsmerkmals sprechen. Im nächsten Schritt sind diese Gesichtspunkte zu gewichten, wobei zwischen einer abstrakten Gewichtung, die dem Aspekt seiner Art nach zukommt, und einer konkreten Gewichtung, gewissermaßen dem Verwirklichungsgrad des Aspekts, zu unterscheiden ist. Und schließlich sind die gewichteten Abwägungsaspekte einander gegenüberzu-

46 Vgl. etwa folgenden Leitsatz aus BGHZ 136, 347: „Besteht ein krasses Missverhältnis zwischen dem Umfang der Haftung und der wirtschaftlichen Leistungsfähigkeit des bürgenden Ehegatten oder Lebenspartners und lässt sich der Verpflichtungsumfang auch nicht im Hinblick auf den Schutz des Gläubigers vor Vermögensverlagerung vom Hauptschuldner auf den Bürgen rechtfertigen, ist der Bürgschaftsvertrag in der Regel gemäß $\mathbb{1 3 8}$ Abs. 1 BGB nichtig. “ (Hervorhebung vom Verf.).

47 Vgl. dazu auch L. Michael, Der allgemeine Gleichheitssatz als Methodennorm komparativer Systeme, Berlin 1997, S. $82 \mathrm{ff}$.

48 Vgl. zur defeasibility näher C. Bäcker, Rules, Principles, and Defeasibility, in: M. Borowski (Hrsg.), On the Nature of Legal Principles, Baden-Baden 2010, S. 79 (81 ff.) sowie die Beiträge in J. Ferrer Beltrán/G. B. Ratti (Hrsg.), The Logic of Legal Requirements: Essays on Defeasibility, Oxford 2012.

49 Zur allgemeinen Struktur von Abwägungsentscheidungen vgl. ausführlich Riehm, Abwägungsentscheidungen (Fn. 9), S. 57 ff.; aus früherer Zeit vgl. auch Hubmann, Wertung und Abwägung (Fn. 18), S. $145 \mathrm{ff}$. 
stellen, um zu ermitteln, welche Gesichtspunkte überwiegen und die Abwägungsentscheidung im engeren Sinne zu treffen. Das sind exakt die gleichen Schritte, die bei jeder Abwägungsentscheidung (im weiteren Sinne) erforderlich sind, ob es sich um eine Beweiswürdigung, eine Abwägung von Argumenten bei der Gesetzesauslegung oder auch die Abwägung verschiedener kollidierender Grundrechte gegeneinander handelt. ${ }^{50}$

\section{Die Auswahl des Abwägungsmaterials}

Bei der Auswahl der berücksichtigungsfähigen Aspekte sind Vorgaben hinsichtlich der zwingend zu berücksichtigenden oder der keinesfalls zu berücksichtigenden Aspekte denkbar. ${ }^{51}$ Zwar gehört es zum Wesen der „Abwägung aller Umstände des Einzelfalls“, dass ex ante kein vollständiger und abschließender Katalog aller denkbaren Abwägungsgesichtspunkte aufgestellt werden kann, weil es gerade der Sinn der Einzelfallabwägung ist, den unwiederholbaren spezifischen Gesichtspunkten des Einzelfalles gerecht zu werden. Gleichwohl kann als Mindestmaß ein Katalog jedenfalls zu berücksichtigender Aspekte aufgestellt werden, bei $\$ 275$ Abs. 2 BGB etwa die im Gesetz bereits erwähnten Größen des Leistungsaufwands des Schuldners und des Leistungsinteresses des Gläubigers, sowie ein etwaiges Vertretenmüssen des Schuldners. In der Literatur findet sich als weiterer stets zu berücksichtigender Abwägungsaspekt eine Risikoübernahme durch den Schuldner, sei es in Gestalt eines konkret übernommenen Aufwands (z. B. eines konkreten Beschaffungsaufwandes) oder eines Aufwandsrisikos. ${ }^{52}$ Auch das Verhalten des Gläubigers, der zu der eingetretenen Leistungserschwerung beigetragen haben kann, ist zu berücksichtigen. ${ }^{53}$

Neben solche positiven Vorgaben für die Auswahl der Abwägungsaspekte treten negative Vorgaben, also Ausschlusskriterien. Solche finden sich zum Einen ausdrücklich in Gesetzen, etwa in $\mathbb{} 46$ Abs. 3 StGB, wonach Umstände, die schon Merkmale des gesetzlichen Tatbestandes sind, bei der Strafzumessung nicht berücksichtigt werden. Zum Anderen ergibt sich das Verbot der Berücksichtigung bestimmter Aspekte auch daraus, dass die berücksichtigungsfähigen Gesichtspunkte im Gesetz abschließend aufgezählt werden: So dürfen nach $\mathbb{1} 1$ Abs. 2 HGB für die Frage, ob ein Gewerbe einen in kaufmännischer Weise eingerichteten Geschäftsbetrieb erfordert, ${ }^{54}$

50 Vgl. dazu Alexy, On Balancing and Subsumption (Fn. 14), S. 440 f.; ders., Die Gewichtsformel, in: J. Jickeli (Hrsg.), Gedächtnisschrift für Jürgen Sonnenschein, Berlin 2003, S. 771 (780 ff.).

51 Neumann, Subsumtion (Fn. 24), S. 332 spricht insoweit zutreffend von „Relevanzregeln“.

52 Vgl. W. Ernst, in: Münchener Kommentar zum Bürgerlichen Gesetzbuch, 6. Aufl., München 2012, \ 275 Rn. 87.

53 Vgl. BGH NJW 2005, 2852 (2854 f.); Ernst (Fn. 52), \275 Rn. 88.

54 Zum Erfordernis einer „Würdigung aller Umstände des Einzelfalles“ zur Ermittlung der „Erforderlichkeit" eines in kaufmännischer Weise eingerichteten Geschäftsbetriebs vgl. nur BGH WM 1960, 935; K. Schmidt, in: Münchener Kommentar zum Handelsgesetzbuch, 2. Aufl., München 2005, $\mathbb{1}$ Rn. 72; C.- W. Canaris, Handelsrecht, 24. Aufl., München 2006, $\$ 3$ Rn. 9; K. J. Hopt, in: Baumbach/ Hopt (Hrsg.), Handelsgesetzbuch, 35. Aufl., München 2012, $\mathbb{1}$ Rn. 23. 
nur Aspekte berücksichtigt werden, die aus Art bzw. Umfang des Gewerbes abgeleitet sind (z. B. Mitarbeiterzahl, Umsatz, Bilanzsumme, Branche u. ä.), nicht aber gänzlich andere Aspekte wie z. B. die Ausbildung oder Geschäftserfahrung des Inhabers, auch wenn diese als allgemeine Argumentationstopoi durchaus nahe lägen, wenn es um die Kaufmannseigenschaft geht. Für die Abwägung nach $\$ 275$ Abs. 2 BGB darf etwa der Vertragspreis keine Rolle spielen, weil er in der Vorschrift nicht erwähnt wird und für ihren Normzweck unerheblich ist. ${ }^{55}$ Auch für die Entscheidung, ob eine Pflichtverletzung „unerheblich“ i.S.v. $\$ 323$ Abs. 5 S. 2 BGB ist und daher das Rücktrittsrecht des Gläubigers ausgeschlossen ist, hat die Rechtsprechung des BGH umfangreiche Kataloge berücksichtigungsfähiger und nicht berücksichtigungsfähiger Aspekte aufgestellt. ${ }^{56}$

Weitere negative Vorgaben finden sich in den allgemeinen Diskriminierungsverboten. So dürften bei $\$ 275$ Abs. 2 BGB das Geschlecht oder die religiöse Überzeugung des Schuldners schon wegen Art. 3 Abs. 3 GG nicht in die Abwägung mit einbezogen werden. ${ }^{57}$ Im Anwendungsbereich des AGG gilt das für die in $\mathbb{} 1$ AGG erwähnten Diskriminierungskriterien, z. B. bei der Bewertung der Gründe für eine Beförderung.

Eine allgemeine negative Vorgabe für die Auswahl des Abwägungsmaterials ist das Verbot sachfremder Erwägungen, das eine Grundbedingung rationaler Rechtsanwendung darstellt. ${ }^{58}$ Zulässig sind also von vornherein - auch bei einer Abwägung aller Umstände des Einzelfalles - nur solche Aspekte, von denen in rationaler Weise behauptet werden kann, dass sie Einfluss auf das Ergebnis haben, also in einen sachlichen Begründungszusammenhang mit dem Abwägungsergebnis gestellt werden können. ${ }^{59}$ Umgekehrt ist das Gericht zugleich gehalten, alle Umstände des Einzelfalls darauf zu überprüfen, ob sie in sachlichem Zusammenhang zum Ergebnis der Abwägungsentscheidung stehen können, und sie ggfs. in seine Entscheidung mit einzubeziehen. Schöpft es den festgestellten Sachverhalt insoweit nicht aus, so ist seine Entscheidung fehlerhaft. ${ }^{60}$ Inwieweit es überdies verpflichtet ist, zusätzliche Umstände festzustellen, um diese der Abwägungsentscheidung zu Grunde legen zu kön-

55 Vgl. S. Lorenz/T. Riehm, Lehrbuch zum neuen Schuldrecht, München 2002, Rn. 306; Ernst (Fn. 52), \$ 275 Rn. 93.

56 Vgl. BGH NJW 2011, 2872 (2873 f., Rn. 18 ff.): Berücksichtigung des Kaufpreises, keine Berücksichtigung der Funktionsbeeinträchtigung durch den Mangel, keine Berücksichtigung früherer Nachbesserungsversuche; BGHZ 167, 19 (23, Rn. 12): Berücksichtigung der Arglist des Schuldners.

57 Näher Riehm, Abwägungsentscheidungen (Fn. 9), S. $59 \mathrm{f}$.

58 Vgl. auch Hubmann, Wertung und Abwägung (Fn. 18), S. 149 mit Hinweis auf Aristoteles, Eudemische Ethik, übersetzt und kommentiert von Franz Dirlmeier, 2. Aufl., Berlin 1969, I 6.

59 Näher Riehm, Abwägungsentscheidungen (Fn. 9), S. 59.

60 Vgl. aus jüngerer Zeit nur BGH WM 2012, 1004 (1007, Rn. 40) zur Unvollständigkeit tatrichterlicher Würdigung zur Feststellung der Verwirkung; BGH NJW 2011, 1442 (1446, Rn. 41 ff.) zur Unvollständigkeit einer Mitverschuldensprüfung; BGH ZIP 2012, 1291 (1296, Rn. 68 ff.) zur unzureichenden Bestimmung einer Entschädigung für immaterielle Schäden. 
nen, ist eine Frage des jeweils geltenden Verfahrensrechts: Eine solche Pflicht besteht nur unter Geltung des Amtsermittlungsgrundsatzes, nicht der Dispositionsmaxime.

\section{Die Gewichtung der Abwägungsaspekte}

Die gefundenen Abwägungsgesichtspunkte sind im Anschluss zu gewichten. Dabei ist zwischen der abstrakten Gewichtung und der konkreten Gewichtung zu unterscheiden.

\section{Abstrakte Gewichtung}

Zunächst ist das Gewicht zu bestimmen, das einem Abwägungsaspekt seiner Art nach zukommt. ${ }^{61}$ Das ist freilich in aller Regel keine Zahl und kein Maß; ${ }^{62}$ aber bereits relative Angaben wie „... ist wichtiger als ...“ oder „... ist gleich wichtig wie ... “ strukturieren die weitere Abwägung. Gleiches gilt für absolute Angaben wie „... kommt nur eingeschränkte Bedeutung zu“63 oder „... kann ... in aller Regel nicht mit dem Argument abgeschnitten werden, ...".64 Ein abstraktes Gewicht kann schließlich auch durch prima-facie-Regeln bestimmt werden, also dadurch, dass bestimmte Aspekte für „,in der Regel“ ausschlaggebend erklärt werden, so dass sie nur in besonderen Ausnahmefällen widerlegt werden können. ${ }^{65}$ Allerdings kann nicht für alle Aspekte ein abstraktes Gewicht ex ante festgelegt werden. Da bei der Abwägung aller Umstände des Einzelfalls die zu berücksichtigenden Gesichtspunkte nicht von vornherein abschließend feststehen, kann deren jeweiliges abstraktes Gewicht konsequenterweise auch nicht abschließend bestimmt werden. „Besondere Umstände“, die nur im konkret zu entscheidenden Einzelfall auftreten, können nicht schon im Voraus abstrakt gewichtet sein.

Soweit ein abstraktes Gewicht bestimmt werden kann, ist dieses definitionsgemäß vom konkreten Einzelfall unabhängig, weil es einem Abwägungsaspekt seiner Art nach zukommt. Dementsprechend ist seine Bestimmung noch Teil der Obersatzbildung; sie ist auch tauglicher Gegenstand des wissenschaftlichen Diskurses, etwa wenn über die Bedeutung und das Gewicht des Vertretenmüssens bei der Abwägung

61 Vgl. zur Unterscheidung zwischen abstrakter und konkreter Gewichtung eingehend Riehm, Abwägungsentscheidungen (Fn. 9), S. $61 \mathrm{ff}$.

62 Denkbar ist das aber schon, etwa bei der Gewichtung verschiedener Indizes zur Ermittlung von Abschreibungskoeffizienten, vgl. BGH RdE 2011, 263 (264, Rn. 10 ff.), die tatsächlich rechnerisch zu erfolgen hat.

63 Vgl. BGH NZM 2010, 174.

64 Vgl. BGH NJW 1996, 3269.

65 Vgl. BGH NJW 2008, 3123 (3125): „... wird die nach $\$ 275$ BGB Abs. 1 S. 1 BGB gebotene Abwägung bei einem Anspruch auf Beseitigung eines grob fahrlässig (und erst recht eines vorsätzlich) errichteten Überbaus in der Regel dazu führen, dass die Einrede zu versagen ist ...". 
nach $\mathbb{2 7 5}$ Abs. 2 BGB diskutiert wird. ${ }^{66}$ Dementsprechend ist es auch tauglicher Gegenstand von Revisionsurteilen bzw. von Leit- und Orientierungssätzen der höchstrichterlichen Rechtsprechung ${ }^{67}$ und der Kommentarliteratur. ${ }^{68}$

\section{Konkrete Gewichtung}

Neben der abstrakten Gewichtung der Abwägungsgesichtspunkte als solche tritt die konkrete Gewichtung, d. h. die Ermittlung des Erfüllungsgrades des jeweiligen Gesichtspunktes im konkreten Fall: Wie groß ist das Leistungsinteresse des Gläubigers, und wie hoch der Leistungsaufwand des Schuldners; gibt es weitere (z. B. immaterielle) Interessen der Parteien, die Naturalleistung zu erhalten bzw. nicht erbringen zu müssen (jeweils zu $\$ 275$ Abs. 2 BGB). Diese Gewichtung ist individuell-konkret, sie bezieht sich also nur auf den konkret zu entscheidenden Fall. Dementsprechend ist sie keiner Verallgemeinerung im Obersatz zugänglich und kann weder Gegenstand höchstrichterlicher Leitsätze noch eines konstruktiven wissenschaftlichen Diskurses sein. Diskutiert werden kann sinnvollerweise allenfalls die Methode zur Ermittlung des konkreten Gewichts, nicht aber das konkrete Gewicht in einem konkreten Fall.

Dementsprechend ist das Gericht bei der Bestimmung des konkreten Gewichts aber auch auf sich alleine gestellt. Wie bei der Beweiswürdigung auch gibt es keine externen Kontrollmaßstäbe, die das Ergebnis seiner Entscheidung abschließend determinieren würden - lediglich das Verfahren der Entscheidung ist vorgegeben. Maßstab für das Entscheidungsergebnis kann - ebenfalls wie bei der Beweiswürdigung ${ }^{69}$ lediglich die persönliche Überzeugung des Gerichts bzw. seiner Mitglieder sein. ${ }^{70}$ Hierin liegt das unausweichliche persönliche Element der Entscheidung selbst. ${ }^{71}$

Die Ermittlung des konkreten Gewichts fällt häufig sogar mit der Beweiswürdigung zusammen, wenn nämlich der Aspekt seinerseits keine normative Bewertung vor-

66 Vgl. dazu A. Helm, Die Einordnung wirtschaftlicher Leistungserschwerungen in das Leistungsstörungsrecht nach der Schuldrechtsreform, Frankfurt a. M. 2005, S. 83, 95; M. Finn, Erfüllungspflicht und Leistungshindernis, Berlin 2007, S. 341 ff.; M. Stürner, Der Grundsatz der Verhältnismäßigkeit im Schuldvertragsrecht, Tübingen 2010, S. 176.

67 Vgl. für ausführliche Abwägungsdirektiven etwa BGH NJW 1996, 3269 f. zu $\$ 633$ Abs. 2 S. 3 BGB a. F.; NJW-RR 2006, 822 (824, Rn. 30 f.) zu $\$ 254$ BGB.

$68 \mathrm{Zu} \ 275$ Abs. 2 BGB vgl. nur Ernst (Fn. 52), $\$ 275$ Rn. $87 \mathrm{ff}$.

69 Vgl. den Wortlaut der $\mathbb{S} \mathbb{S} 286$ ZPO, 261 StPO; es ist jedoch umstritten, inwieweit diese Überzeugung - wie hier vertreten - tatsächlich subjektiv zu verstehen ist, vgl. dazu Riehm, Abwägungsentscheidungen (Fn. 9), S. 82 ff. m. w. N. und einer Synthese von objektiver und subjektiver Beweismaßtheorie. Kritisch zur Rolle der Überzeugung etwa Freund, Tatsachenfeststellung (Fn. 3), S. 46 ff., 56 ff.

70 Vgl. zur Bedeutung der Überzeugung des Entscheiders bei Abwägungsentscheidungen eingehend Riehm, Abwägungsentscheidungen (Fn. 9), S. 71, 77 ff.; ferner J. Lege, Pragmatismus und Jurisprudenz, Tübingen 1999, S. 487 ff.; A. Engländer, Der Begriff der freien Überzeugung in $\mathbb{} 261 \mathrm{StPO}$ im Lichte der Erkenntnistheorie, in: R. Alexy (Hrsg.), Juristische Grundlagenforschung, Stuttgart 2005, S. 85 ff.; J. Hänni, Vom Gefühl am Grund der Rechtsfindung, Berlin 2011, S. 91, 106 ff.; s. aus rechtssoziologischer Sicht dazu M. Kronenberger, Der Parasit der Überzeugungsbildung, Berlin 2010, S. $62 \mathrm{ff} ., 81 \mathrm{ff}$.

71 Vgl. dazu etwa K. Engisch, Wahrheit und Richtigkeit im juristischen Denken, München 1963, S. 309 f.; T. Osterkamp, Juristische Gerechtigkeit, Tübingen 2004, S. 95. 
aussetzt, sondern lediglich auf ein empirisches Faktum verweist. So können etwa der Leistungsaufwand des Schuldners oder das monetäre Leistungsinteresse des Gläubigers im Rahmen des $\$ 275$ Abs. 2 BGB im Wege der Beweisaufnahme, z. B. mit Hilfe eines Sachverständigengutachtens, geklärt werden. Das ist aber nicht zwangsläufig der Fall: Ob und ggfs. in welchem Maße der Schuldner die Leistungserschwerung zu vertreten hat (vgl. $\$ 275$ Abs. 2 S. 2 BGB), ist keine Frage, die einem empirischen Beweis unmittelbar zugänglich wäre. Vielmehr kann das Gericht sie nur anhand einer rechtlichen Bewertung beantworten, das konkrete Gewicht dieses Abwägungsgesichtspunktes also nur durch Wertung bestimmen. Dort zeigt sich der Unterschied zwischen der Beweiswürdigung und der Einzelfallabwägung.

\section{Die Ermittlung des Ergebnisses}

Sind die widerstreitenden Abwägungsgesichtspunkte gewichtet, so müssen sie einander gegenübergestellt werden, um zu ermitteln, welche Gesichtspunkte überwiegen. Bei Abwägungen, die ein Ergebnis auf einer kontinuierlichen Skala ergeben sollen, folgt dieses bereits unmittelbar aus der Gegenüberstellung der widerstreitenden Aspekte. Das deutlichste Beispiel einer solchen Abwägung ist die zwischen den Verursachungsanteilen von Schädiger und Geschädigtem nach $\$ 254$ Abs. 1 BGB, wo sich das Gericht eine Überzeugung vom Verhältnis der jeweiligen Verursachungsbeiträge bilden muss, um hieraus dann unmittelbar die Haftungsquoten abzuleiten.

Anders liegt es dagegen bei Entscheidungen, die auf eine binäre Entscheidungsalternative hinauslaufen, insbesondere auf das Vorliegen oder Nichtvorliegen eines normativen Tatbestandsmerkmals: Hier muss das Ergebnis (ja oder nein) aus dem Verhältnis der Gesichtspunkte für und wider das Vorliegen abgeleitet werden. Dafür ist es erforderlich, eine Schwelle zu bestimmen, welche die Gesichtspunkte für das Vorliegen des Tatbestandsmerkmals erreichen müssen, um das Subsumtionsergebnis „ja“ auszulösen. Eine solche strukturelle Kategorie ist aus dem Recht der Beweiswürdigung bekannt und heißt dort Beweismaß. ${ }^{72}$ Analog hierzu soll die Schwelle für die Einzelfallwürdigung im Folgenden Wertungsmaß genannt werden. ${ }^{73}$

Häufig tritt diese Schwelle nicht gesondert ins Bewusstsein, weil es genügt festzustellen, ob die (gewichteten) Gesichtspunkte für die eine Seite die Gesichtspunkte für die andere Seite (schlicht) überwiegen, ob also aus der Sicht des Entscheiders „die besseren Gründe " für eine Seite sprechen. Manche Normen erfordern darüber hinaus jedoch ein qualifiziertes Überwiegen: So verlangt $\$ 275$ Abs. 2 BGB ein „grobes Missverhältnis“ zwischen Leistungsaufwand des Schuldners und Leistungsinteresse

72 Vgl. hierzu grundlegend B. M. Maassen, Beweismaßprobleme im Schadensersatzprozess, Köln u. a. 1975; M. Huber, Das Beweismaß im Zivilprozeß, Köln u. a. 1983; D. Leipold, Beweismaß und Beweislast im Zivilprozeß, Berlin 1985 sowie die Diskussion zusammenfassend Riehm, Abwägungsentscheidungen (Fn. 9), S. 82 ff.

73 Vgl. eingehend Riehm, Abwägungsentscheidungen (Fn. 9), S. 92 ff. 
des Gläubigers. ${ }^{74}$ Ähnlich setzen die $\mathbb{\$} \$ 319$ Abs. 1 S. 1, 660 Abs. 1 S. 2 Hs. 1 oder 2048 S. 3 BGB voraus, dass die Bestimmung der Leistung, die Verteilung einer ausgelobten Summe oder die Teilung des Nachlasses durch einen Dritten „offenbar unbillig“ ist - hier ist der Unterschied zur (einfachen) „Unbilligkeit“ in $\mathbb{} 315$ Abs. 3 BGB deutlich zu sehen. ${ }^{75}$ Anders als manche Autoren meinen ${ }^{76}$ handelt es sich hierbei nicht um eine Erhöhung des Beweismaßes, sondern um eine solche des Wertungsmaßes, weil die zu treffende Entscheidung eben keine Beweiswürdigung von empirischen Tatsachen ist, sondern eine rechtliche Wertungsentscheidung über die Billigkeit bzw. Unbilligkeit.

Im vorstehenden Text wurde bereits angedeutet, dass das Ergebnis der Abwägung nur nach der Überzeugung des Gerichts festgestellt werden kann. Wie bei der konkreten Gewichtung der Abwägungsaspekte ${ }^{77}$ fehlt es für die wertende Gegenüberstellung der gewichteten Aspekte an objektiven, intersubjektiv nachvollziehbaren Maßstäben, die eine Determination (und inhaltliche Kontrolle) des Abwägungsergebnisses ermöglichen würden. ${ }^{78}$ In Ermangelung derartiger objektiver Maßstäbe verbleibt als einzige Möglichkeit der Verweis auf die richterliche Eigenwertung. Dabei ist zu betonen, dass es nicht um die Ausweitung richterlichen Dezisionismus' geht, sondern umgekehrt gerade um dessen Begrenzung auf diejenigen Entscheidungsschritte, in denen es an objektiven inhaltlichen Maßstäben fehlt - um im Gegenzug die übrigen Entscheidungsschritte einer objektiven Falsifikation anhand der oben ausgeführten Abwägungsregeln zugänglich zu halten.

\section{Die Wertungslast}

Die Parallelen zur Beweiswürdigung erschöpfen sich nicht in der Existenz einer Kategorie des Wertungsmaßes, die praktisch eine verhältnismäßig geringe Bedeutung hat. Vielmehr erstrecken sie sich auf die wesentlich bedeutsamere Kategorie der Beweislast, als deren Pendant bei der rechtlichen Einzelfallabwägung hier die Kategorie der Wertungslast eingeführt werden soll. ${ }^{79}$ Die Wertungslast entscheidet demnach, welche Entscheidung zu treffen ist, wenn das Gericht bei der rechtlichen Würdigung aller Umstände des Einzelfalles zu keiner hinreichenden Überzeugung gelangt, die Einzelfallabwägung selbst also ohne Ergebnis bleibt (non liquet).

74 Vgl. dazu näher Lorenz/Riehm, Lehrbuch zum neuen Schuldrecht (Fn. 55), Rn. 309 f.; T. Riehm, Der Grundsatz der Naturalerfüllung, i. V. für 2013, $\$ 7$ IV 2 e.

75 Vgl. zur Unterscheidung beider Maßstäbe nur M. Würdinger, in: Münchener Kommentar zum Bürgerlichen Gesetzbuch (Fn. 52), $\$ 319$ Rn. 6.

76 Vgl. H. Prütting, Gegenwartsprobleme der Beweislast, München 1983, S. 83; H.-J. Musielak/M. Stadler, Grundfragen des Beweisrechts, München 1984, Rn. 148.

77 Vgl. dazu soeben C.II.2.

78 Vgl. eingehend Riehm, Abwägungsentscheidungen (Fn. 9), S. 77 ff.

79 Eingehend Riehm, Abwägungsentscheidungen (Fn. 9), S. 122 ff. 


\section{Möglichkeit eines non liquet}

Nun wird allerdings von der ganz überwiegenden Auffassung bestritten, dass es in Rechtsfragen - und damit auch bei der rechtlichen Würdigung aller Umstände des Einzelfalles - ein non liquet geben kann. ${ }^{80}$ Vielfach heißt es, das Tatgericht müsse sich in Rechtsfragen „zu einer bestimmten Entscheidung durchringen“; ${ }^{81}$ Rechtsfragen seien „einer Beweislastentscheidung nicht zugänglich “. 82

Diese Auffassung ignoriert allerdings die entscheidungspsychologische Realität bei Abwägungsentscheidungen wie der Einzelfallwürdigung: Wenn das Ziel der Würdigung aller Umstände des Einzelfalles die persönliche Überzeugung des Entscheiders (bzw. der Mehrheit der Mitglieder eines Entscheidungsgremiums, etwa eines Spruchkörpers) ist, dann kann die Situation eintreten, dass der Entscheider nicht zu einer hinreichend sicheren Überzeugung vom Überwiegen der Gründe für eine Seite gelangt. ${ }^{83}$ Das kann an (subjektiven) Unsicherheiten bei der Bewertung einzelner Aspekte liegen, am Verfehlen eines erhöhten Wertungsmaßes, und bei Gremienentscheidungen schließlich an einem Abstimmungspatt. Jeder praktisch tätige Jurist kennt die Situation, dass er von zwei Alternativen beide für gleichermaßen überzeugend (oder gleichermaßen wenig überzeugend) hält und heute zur einen und morgen zur anderen Alternative tendiert. Wer in einer solchen Situation vom Entscheider verlangt, er müsse sich zu einer Entscheidung durchringen verlangt Unmögliches: Die subjektive Überzeugung ist das „durch psychische Vorgänge hervorgerufene Empfinden einer Person, welches sie veranlasst, ein [...] Urteil als für sich gültig anzuerkennen ", 84 und ein solches Empfinden kann nicht erzwungen werden; es stellt sich am Ende des Abwägungsvorgangs ein - oder eben nicht.

Wollte man in dieser Situation gleichwohl vom Entscheider verlangen, seine Entscheidung aufgrund einer Abwägung zu treffen, so hätte dieser nur die Möglichkeit,

80 Vgl. K. Schwindel, Das non liquet in der Tatfrage, Diss. München 1963, S. 13 ff.; L. Rosenberg, Die Beweislast, 5. Aufl., München/Berlin 1965, S. 9; Prütting, Gegenwartsprobleme (Fn. 76), S. 122; D. Leipold, in: Stein/Jonas (Hrsg.), Zivilprozessordnung, 22. Aufl., Tübingen 2008, $\$ 286$ Rn. $49 ; H$. Prütting, in: Rauscher/Wax (Hrsg.), Münchener Kommentar zur Zivilprozessordnung, 3. Aufl., München 2008, \$286 Rn. 96; wie hier dagegen F. Bydlinski, Hauptpositionen zum Richterrecht, JZ 1985, S. 149 (156 f.); C.-W. Canaris, Richtigkeit und Eigenwertung in der richterlichen Rechtsfindung, in: Universität Graz (Hrsg.), Grazer Universitätsreden, Bd. 50, Graz 1993, S. 23 (30); P. Krebs, Die Begründungslast, AcP 195 (1995), S. 171 (173 ff.); J. Prölss, Die Verteilung der Begründungslast als Inhalt von Entscheidungsregeln bei Rechtsanwendungszweifeln, in: M. P. Stathopulos (Hrsg.), FS Georgiades, München 2006, S. 1063 (1065); W. Fleck, Begriff und Funktion der „Billigkeit“ bei $\$ 284$ BGB, JZ 2009, S. 1045 (1053).

81 Rosenberg, Beweislast (Fn. 80), S. 9; Prütting, Gegenwartsprobleme (Fn. 76), S. 122; ähnlich RG JW 1915, 650 (zur Auslegung einer Willenserklärung).

82 BGH NJW 1987, 901 (zur Auslegung von Willenserklärungen); ebenso Rosenberg, Beweislast (Fn. 80), S. 9.

83 Vgl. Riehm, Abwägungsentscheidungen (Fn. 9), S. 115 f.

84 Vgl. R. Greger, Beweis und Wahrscheinlichkeit, Köln u. a. 1978, S. 20; ähnlich bereits I. Kant, Kritik der reinen Vernunft, 1787, B 850; s. ferner E. Döhring, Die Erforschung des Sachverhalts im Prozess, Berlin 1964, S. 463; G. Bohne, Zur Psychologie der richterlichen Überzeugungsbildung, Köln 1967, S. 10 ff.; Maassen, Beweismaßprobleme (Fn. 72), S. 29. 
die Entscheidung ganz zu verweigern, oder eine subjektiv willkürliche Entscheidung zu treffen, die nicht auf seiner Überzeugung beruht. Die erste Möglichkeit widerspräche dem Verbot der Rechtsverweigerung ${ }^{85}$ die zweite dem rechtsstaatlichen Gebot rationaler Rechtsfindung ${ }^{86}$ und würde zudem das heuristische Potenzial von Wertungslastregeln nicht nutzen. ${ }^{87}$

Damit soll freilich nicht einem verfrühten Ausweichen auf Wertungslastregeln das Wort geredet werden. Es bleibt dabei, dass die Gerichte - wie auch bei der Beweiswürdigung ${ }^{88}$ - auf Lastregeln erst zurückgreifen dürfen, wenn sie den Sachverhalt vollständig ausgeschöpft und gewürdigt haben, und gleichwohl nicht zu einer Überzeugung gelangt sind. Diese Situation dürfte hauptsächlich in Fällen auftreten, in denen das Wertungsmaß erhöht ist, da eine Überzeugung vom „einfachen“ Überwiegen der Aspekte für eine Seite in den weitaus meisten Fällen zu erlangen sein wird.

\section{Funktionen der Wertungslast}

Die Kategorie der Wertungslast dient nicht nur dazu, für die Fälle eines non liquet in Bewertungsfragen zu einer Entscheidung zu gelangen, sondern - logisch untrennbar damit verbunden - auch dazu, die Frageperspektive festzulegen, aus welcher die Einzelfallabwägung vorgenommen wird: Trägt derjenige die Wertungslast, der sich auf das Vorliegen eines normativen Tatbestandsmerkmals (z. B. der „Unangemessenheit“) beruft, so muss das Gericht nicht zwischen den Alternativen „unangemessen“ oder „angemessen“ seine Überzeugung bilden, sondern allein die Frage beantworten, ob es von der „Unangemessenheit“ überzeugt ist oder nicht. Gelangt es nicht zu der Überzeugung, dass etwas „unangemessen“ ist, so ist es unerheblich, ob es zugleich zu der Überzeugung gelangt, dass es ,angemessen“ ist - das Tatbestandsmerkmal ist in jedem Fall abzulehnen. Implizit wird dadurch das non liquet, in welchem das Gericht weder von der „Angemessenheit“ noch von der „Unangemessenheit" überzeugt ist, faktisch der „Angemessenheit“" zugeschlagen, also die Wertungslast verteilt. Diese Reduktion der Perspektive von der bipolaren Frage ,angemessen oder unangemessen?“ auf die unipolare Frage „unangemessen?“ ist eine praktisch unentbehrliche Funktion der Wertungslastverteilung und in dieser logisch enthalten.

Damit wird zugleich deutlich, dass die Wertungslastregeln auch einen materiellen Gehalt haben, indem sie nämlich eine ,intendierte Entscheidung“ vorgeben, die immer dann zu treffen ist, wenn das Gericht nicht vom Gegenteil überzeugt ist - also ein normatives Regel-Ausnahme-Verhältnis festlegen. Deutlich wird das etwa an der

85 Vgl. dazu BVerfGE 84, 212 (227); E. Schumann, Das Rechtsverweigerungsverbot, ZZP 81 (1968), S. 79 (80); C. W. Hergenröder, Zivilprozessuale Grundlagen richterlicher Rechtsfortbildung, Tübingen 1995, S. 168 ff.; J. Neuner, Die Rechtsfindung contra legem, 2. Aufl., München 2005, S. 53 f.

86 Vgl. auch Canaris, Lücken (Fn. 38), S. 175.

87 S. dazu näher Riehm, Abwägungsentscheidungen (Fn. 9), S. 130 ff.

88 Vgl. H. J. Musielak, Die Grundlagen der Beweislast im Zivilprozeß, Berlin u. a. 1975, S. 3, 19; Prütting (Fn. 80), $\$ 286$ Rn. 93. 
Vorschrift des $\mathbb{3} 323$ Abs. 5 S. 2 BGB: Danach ist der Rücktritt im Falle einer mangelhaften Leistung ausgeschlossen, wenn die Pflichtverletzung „unerheblich“ ist. ${ }^{89}$ Durch die negative Formulierung wird dem Rücktrittsgegner die Wertungslast für die „Unerheblichkeit“ der Pflichtverletzung zugewiesen; kann sich das Gericht nicht von der „Unerheblichkeit“ überzeugen, so bleibt es bei dem von $\$ 323$ Abs. 1 BGB eingeräumten Rücktrittsrecht - ohne dass es darauf ankäme, ob das Gericht von der „Erheblichkeit ${ }^{“ 90}$ der Pflichtverletzung überzeugt ist. ${ }^{91}$ Aus dieser Wertungslastverteilung folgt zugleich ein „Grundsatz der Gesamtabwicklung“, d. h. der normative Regelfall im Falle einer Schlechtleistung ist das Bestehen eines Rücktrittsrechts, und nur im Ausnahmefall ist das Rücktrittsrecht ausgeschlossen. ${ }^{92}$

Eine weitere Funktion der Wertungslast liegt schließlich in ihrer Reflexwirkung auf die faktische Vortragslast: Wer sich auf die „Unangemessenheit“ berufen muss, also insoweit die Wertungslast trägt, wird in seinem eigenen Interesse auch die dafür erforderlichen Tatsachen vortragen. Nur unter Verfahrensordnungen mit Dispositionsmaxime folgt daraus auch eine rechtliche Vortragslast in dem Sinne, dass nicht vorgetragene Elemente keine Berücksichtigung finden. Das allerdings ist eine Frage der Tatsachenfeststellung, nicht der rechtlichen Einzelfallabwägung. Unter Geltung des Amtsermittlungsgrundsatzes (etwa im Verwaltungsprozess oder in der freiwilligen Gerichtsbarkeit) kommt eine solche rechtliche Vortragslast ohnehin nicht in Betracht. Gleichwohl kann es auf der Grundlage des von Amts wegen festgestellten Sachverhalts zu einem non liquet kommen, das nach den Grundsätzen der Wertungslast aufzulösen ist. ${ }^{93}$

\section{Regelungen der Wertungslast}

Dass die Kategorie der Wertungslast - und damit die Möglichkeit eines non liquet bei der rechtlichen Einzelfallwürdigung - existiert, zeigen zahlreiche gesetzliche Regelungen aus den verschiedensten Rechtsgebieten, in welchen der Gesetzgeber eine solche Kategorie implizit voraussetzt.

89 Zum Erfordernis einer Einzelfallabwägung für die Subsumtion unter das Merkmal „unerheblich“ vgl. BGH NJW 2011, 2872 (2873 f., Rn. 7, 18 ff.).

90 Gerade das Begriffspaar „unerheblich“ - „erheblich“ (vgl. etwa $\$ 651 \mathrm{f}$ Abs. 2 BGB für einen Anspruch auf immaterielle Schäden, wenn die Reise „erheblich“ beeinträchtigt wird) zeigt, dass es zwischen diesen beiden Polen auch einen Bereich „,normaler“ Pflichtverletzungen gibt, der weder dem einen noch dem anderen Pol zuzuordnen ist, also einem non liquet entspricht.

91 Ungenau daher Ernst (Fn. 52), \323 Rn. 243 ff., der auf die Erheblichkeit der Pflichtverletzung abstellt; richtig demgegenüber BGHZ 136, 94 (98f.) zur Vorgängernorm des $\$ 459$ Abs. 1 S. 2 BGB a. F., der allein die „Unerheblichkeit“ eines Sachmangels prüft, ohne nach seiner „Erheblichkeit“ zu fragen.

92 Vgl. näher H. C. Grigoleit/T. Riehm, Grenzen der Gleichstellung von Zuwenig-Leistung und Sachmangel, ZGS 2002, S. 115 (116); B. Heiderhoff/F. Skamel, Teilleistung im Kaufrecht, JZ 2006, S. 383 (384).

93 Vgl. dazu Riehm, Abwägungsentscheidungen (Fn. 9), S. 127 f. 


\section{a) Die Vermutung des Vorliegens eines Handelsgewerbes ( $\$ 1$ Abs. 2 HGB)}

So bestimmt etwa $\mathbb{1} 1$ Abs. 2 HGB, dass jeder Gewerbebetrieb als Handelsgewerbe anzusehen ist, „es sei denn, dass das Unternehmen nach Art oder Umfang einen in kaufmännischer Weise eingerichteten Geschäftsbetrieb nicht erfordert.“ Dieser Satz ist aufgrund seiner negativen Formulierung zunächst als Regelung der Darlegungsund Beweislast zu verstehen. ${ }^{94}$ Als solche könnte sie im Prozess jedoch kaum praktische Bedeutung entfalten, denn Art und Umfang des Unternehmens - zu messen an Indikatoren wie Branche, Personalstärke, Zahl und Art der getätigten Geschäfte, Umsatz oder Bilanzsumme ${ }^{95}$ - werden regelmäßig entweder unstreitig oder zumindest vom Gewerbetreibenden liquide beweisbar sein. Eine Regelung der Beweislast im Sinne der Auflösung eines non liquet in der Tatfrage ist daher einerseits kaum nötig und würde andererseits das eigentliche Regelungsziel, nämlich die Eindämmung der mit dem Abstellen auf das unscharfe Kriterium der „Erforderlichkeit eines in kaufmännischer Weise eingerichteten Geschäftsbetriebs“ zwangsläufig verbundenen Rechtsunsicherheit, ${ }^{96}$ nicht optimal erreichen. Denn die Rechtsunsicherheit droht nicht primär wegen der tatsächlichen Probleme bei der Feststellung von Art oder Umfang des Unternehmens, sondern vor allem wegen der Unschärfe des Begriffes der „Erforderlichkeit“ eines in kaufmännischer Weise eingerichteten Geschäftsbetriebs, dessen Anwendung eine Abwägung aller Umstände des Einzelfalles erforderlich macht. ${ }^{97}$ Diese Aufgabe kann die Norm nur erfüllen, wenn sie (auch) als Wertungslastregel für die Frage verstanden wird, ob in einer bestimmten (unstreitigen oder festgestellten) Sachverhaltskonstellation ein in kaufmännischer Weise eingerichteter Geschäftsbetrieb erforderlich ist. ${ }^{98}$ Versteht man $\ 1$ Abs. 2 HGB auf diese Art, so trägt der Gewerbetreibende im Prozess nicht nur das (praktisch vernachlässigbare) Risiko, dass er die Tatsachen zu Art und Umfang seines Gewerbes nicht beweisen kann, sondern darüber hinaus das praktisch wesentlich größere Risiko, dass das Gericht anhand der von ihm vorgelegten Tatsachen nicht die (rechtliche) Überzeugung gewinnen kann, ein in kaufmännischer Weise eingerichteter Geschäftsbetrieb sei nach Art und Umfang seines Gewerbes nicht erforderlich.

\section{b) Die Regelbeispiele des $\S 307$ Abs. 2 BGB}

Ein weiterer Fall einer Wertungslastregel findet sich in $\$ 307$ Abs. 2 BGB. Nach dieser Vorschrift ist eine unangemessene Benachteiligung durch eine Klausel in Allgemeinen Geschäftsbedingungen „im Zweifel“ anzunehmen, wenn diese entweder mit wesentlichen Grundgedanken der gesetzlichen Regelung nicht zu vereinbaren ist oder die

94 Vgl. nur Canaris, Handelsrecht (Fn. 54), $\$ 3$ Rn. 11.

95 Vgl. Canaris, Handelsrecht (Fn. 54), $\mathbb{3}$ Rn. 9.

96 Vgl. Regierungsbegründung zum Handelsrechtsreformgesetz, BT-Drs. 13/8444, S. $26,48$.

97 Vgl. nur BGH WM 1960, 935; Canaris, Handelsrecht (Fn. 54), $\$ 3$ Rn. 9; Hopt (Fn. 54), $\$ 1$ Rn. 23.

98 Der Sache nach ebenso Canaris, Handelsrecht (Fn. 54), $\$ 3$ Rn. 12; vgl. auch P. Krebs, Reform oder Revolution?, DB 1996, S. 2013 (2018). 
Erreichung des Vertragszwecks gefährdet. Über die Bedeutung der Formulierung „im Zweifel" herrscht Streit: 99 Teilweise wird vertreten, diese Formulierung sei schon deswegen ohne Aussagewert, weil es Zweifel bei der Rechtsfrage, ob eine Klausel angemessen oder unangemessen sei, ohnehin nicht geben dürfe, und Tatsachenfragen im Rahmen der Inhaltskontrolle kaum je zweifelhaft seien. ${ }^{100}$ Nach der zutreffenden Gegenansicht ist $\mathbb{3} 307$ Abs. 2 BGB dagegen als Wertungslastregel zu verstehen. ${ }^{101}$ Nur dieses systematische Verständnis des $\$ 307$ Abs. 2 BGB als Umkehr der Wertungslast vermag der Vorschrift eine gegenüber $\$ 307$ Abs. 1 BGB eigenständige Funktion zuzuweisen. Denn $\mathbb{3} 307$ Abs. 1 BGB erfordert ohnehin eine umfassende Interessenabwägung, die auch dann erforderlich ist, wenn ein Tatbestand des $\$ 307$ Abs. 2 BGB erfüllt ist - nur eben gewissermaßen mit umgekehrtem Vorzeichen. ${ }^{102}$ Die Funktion als Wertungslastverteilung bringt die Formulierung „eine unangemessene Benachteiligung ist im Zweifel anzunehmen " auch deutlich zum Ausdruck, denn gerade um solche Zweifel in der rechtlichen Bewertungsfrage und die Verteilung der Wertungslast in diesen Zweifelsfällen geht es hier. Wenn dagegen eingewendet wird, bei Rechtsfragen gebe es keine „Beweislast“, und eine „Wertungslast“ oder „Argumentationslast" sei dem geltenden Recht fremd, weil es Zweifel in Rechtsfragen eben nicht geben dürfe ${ }^{103}$ so handelt es sich um einen klassischen Zirkelschluss, der durch die soeben dargelegte Normstruktur des $\$ 307$ BGB selbst widerlegt wird; zudem werden dabei die weiteren hier angeführten Gegenbeispiele ignoriert, die alle eine gesetzliche Wertungslastverteilung vorsehen.

\section{c) Die Auslegungsregel ,in dubio contra proferentem“ für AGB ( $\$ 305$ c Abs. 2 BGB)}

Ein anderes Beispiel - wiederum aus dem AGB-Recht - bildet die Auslegungsregel für Allgemeine Geschäftsbedingungen in $\$ 305$ c Abs. 2 BGB. Zweifel bei der Auslegung von AGB gehen danach zu Lasten des Verwenders. Nun handelt es sich bei der Auslegung von Willenserklärungen um eine Rechtsfrage, die ebenfalls eine Abwägung aller Umstände des Einzelfalls - bzw. bei AGB eine Abwägung aller objektiver Auslegungsindizien - erfordert. ${ }^{104}$ In ständiger Rechtsprechung akzeptiert es der BGH allerdings nicht, dass die Instanzgerichte bei der Auslegung nicht zu einem ein-

99 Vgl. zum Streitstand C.-W. Canaris, Die AGB-rechtliche Leitbildfunktion des neuen Leistungsstörungsrechts, in: M. Habersack (Hrsg.), FS Ulmer, Berlin 2003, S. 1075 f.

100 Vgl. etwa M. Coester, in: Staudinger (Hrsg.), BGB, Berlin 2006, \$ 307 Rn. 222 ff., der das Merkmal „im Zweifel“ konsequenterweise für bedeutungslos hält, vgl. ebd. Rn. 226 a. E.; ebenso M. Stoffels, AGB-Recht, 2. Aufl., München 2009, Rn. 500.

101 So in der Sache (mit z. T. abweichender Terminologie „Argumentationslastregel“) Krebs, Die Begründungslast (Fn. 80), S. 174, 180 f.; Canaris, AGB-rechtliche Leitbildfunktion (Fn. 99), S. 1078 ff.; Prölss, Begründungslast (Fn. 80), S. 1069 f.; der Sache nach wohl auch BGHZ 153, 148 (155): ,Vermutung der unangemessenen Benachteiligung “.

102 Vgl. BGHZ 82, 238 (240 f.); BGHZ 133, 10 (15 f.); BGHZ 153, 344 (349 ff.); Canaris, AGB-rechtliche Leitbildfunktion (Fn. 99), S. $1079 \mathrm{ff}$.

103 Vgl. Coester (Fn. 100), \$ 307 Rn. 222 f.

104 Vgl. Riehm, Abwägungsentscheidungen (Fn. 9), S. 119 ff. m. w. N. 
deutigen (Abwägungs-)Ergebnis gelangen. ${ }^{105}$ Die regelmäßig wiederkehrenden Revisionsentscheidungen zu dieser Frage zeigen aber, dass die Praxis der Instanzgerichte immer wieder vor der entscheidungspsychologischen Realität steht, dass am Ende der Abwägung mehrere Auslegungsvarianten einer Willenserklärung gleichermaßen überzeugend erscheinen. ${ }^{106}$ Diese Situation greift die Zweifelsregelung des $\mathbb{S} 305 \mathrm{c}$ Abs. 2 BGB im Hinblick auf AGB auf, indem sie für genau diesen Fall eine Entscheidung zu Lasten des Verwenders vorgibt. Die revisionsgerichtliche Rechtsprechung ist in sich widersprüchlich, wenn sie einerseits für die Anwendung des $\mathbb{S} 305 \mathrm{c}$ Abs. 2 BGB verlangt, dass „Zweifel“ im Sinne dieser Vorschrift nur vorliegen, wenn nach Anwendung aller Auslegungskriterien und Ausschöpfung aller Auslegungselemente - also am Ende der Abwägung - noch mehrere Auslegungsvarianten vertretbar sind und zwischen diesen ein nicht behebbarer Zweifel bleibt, ${ }^{107}$ andererseits aber bei der Auslegung einfacher Willenserklärungen die Möglichkeit eines solchen nicht behebbaren Zweifels leugnet. ${ }^{108}$ Umgekehrt ist aus $\mathbb{3} 305$ c Abs. 2 BGB, der einen solchen Zweifel tatbestandlich voraussetzt, zu folgern, dass es einen solchen unbehebbaren Zweifel in Auslegungsfragen geben kann (und darf), und dass dieser mit Hilfe von Wertungslastregeln zu beheben ist. Das gilt nicht nur für Allgemeine Geschäftsbedingungen, sondern auch für einfache Willenserklärungen - wenn auch mit von $\mathbb{S} 305$ c Abs. 2 BGB abweichender Wertungslastverteilung. ${ }^{109}$

\section{d) Das intendierte Verwaltungsermessen}

Auch im öffentlichen Recht verwendet der Gesetzgeber implizite Verteilungen der Wertungslast. Besonders offensichtlich ist dies bei dem sog. intendierten Ermessen: ${ }^{110}$ Hiervon spricht das BVerwG, wenn dem Gesetz zu entnehmen ist, welches Ergebnis einer Ermessensentscheidung für den Normalfall gewollt ist. ${ }^{111}$ Von diesem gewollten Ergebnis ist nur abzuweichen, wenn besondere Umstände vorliegen, die eine solche Abweichung fordern. Zumindest missverständlich ist es allerdings, wenn formuliert wird, ohne das Vorliegen besonderer Umstände müsse die Behörde keine

105 Vgl. BGH NJW-RR 1989, 1282; NJW 1987, 901; NJW 1984, 721 f.; BGHZ 20, 109 (110 f.); ebenso bereits RG JW 1915, 650; JW 1927, 514; R. Pohle, Auslegung und Beweislast, MDR 1951, S. $91 \mathrm{ff}$.

106 Vgl. auch E. Krönig, Die Bedeutung der Beweislast bei Auslegungsfragen (ein zu Unrecht verkannter Gesichtspunkt), MDR 1950, S. 664 ff.; ders., Erwiderung auf Pohle, MDR 1951, S. 92.

107 Vgl. nur BGHZ 91, 98 (103); BGHZ 112, 65 (68 f.); BGH NJW 1997, 3434 (3435); NJW 2002, 3232 (3233); NJW-RR 2006, 338 (339).

108 Soeben Fn. 105.

109 Vgl. zur Beweislast für die Auslegung von Willenserklärungen - übertragbar auf die Wertungslast eingehend B. Gsell, Die Beweislast für den Inhalt der vertraglichen Einigung, AcP 203 (2003), S. $119 \mathrm{ff}$.

110 S. die Darstellung bei S. Detterbeck, Allgemeines Verwaltungsrecht, 10. Aufl., München 2012, Rn. 322 ff. sowie eingehend U. Volkmann, Das „intendierte“ Verwaltungsermessen, DÖV 1996, S. 282 ff.; Borowski, Intendiertes Ermessen (Fn. 9); J. Beuermann, Intendiertes Ermessen, Frankfurt a. M. 2002; zur Einordnung der Ermessensausübung als Abwägungsentscheidung vgl. Riehm, Abwägungsentscheidungen (Fn. 9), S. 46 ff.

111 Vgl. BVerwGE 72, 1 (6); 91, 82 (90). 
Ermessenserwägungen anstellen, bzw. es bedürfe „keiner Abwägung des ,Für und Wider'“. ${ }^{112}$ Denn bereits die Feststellung, ob die Umstände des Falles „besondere“ sind, die eine Abweichung vom Regelfall erfordern, kann nur durch deren Gewichtung und Vergleich mit dem „Normalfall“ erfolgen, also durch eine Abwägungsentscheidung.

Das Erfordernis besonderer Umstände für die Abweichung vom Regelfall begründet dabei zugleich regelmäßig eine Erhöhung des Wertungsmaßes: Nicht jede Überzeugung vom (einfachen) Überwiegen der Gesichtspunkte für eine Seite kann eine Abweichung von der intendierten Entscheidung begründen, sondern nur die Überzeugung vom erheblichen Überwiegen. Mit anderen Worten müssen die Gründe, die den Gesetzgeber dazu bewogen haben, im Regelfall eine bestimmte Entscheidung vorzugeben, widerlegt sein. Je gewichtiger diese Gründe sind, desto höher sind die Anforderungen an die „Besonderheit“ der Umstände, desto höher ist also das zu erfüllende Wertungsmaß.

Diese Entscheidungsstruktur beim intendierten Ermessen zeigt, dass gleichwohl eine Abwägung stattfindet, wobei die gleichen Gesichtspunkte zu berücksichtigen sind, als wäre die Ermessensentscheidung nicht „intendiert“. Ist der Entscheider davon überzeugt, dass die Gesichtspunkte in der einen oder anderen Richtung hinreichend überwiegen, so entscheidet er aufgrund einer Abwägung aller Umstände des Einzelfalls, ohne dass es hierfür darauf ankommt, dass die Entscheidung „intendiert“ ist. Insofern ist die neuere Formulierung der Verwaltungsgerichtsbarkeit präziser, wonach sich im Regelfall „das Ergebnis der Abwägung von selbst verstehe“. ${ }^{113}$ Die strukturelle Besonderheit des intendierten Ermessens zeigt sich hierbei in zwei Aspekten: Zum einen wird die Entscheidung für ein non liquet vorgegeben, das insbesondere wegen des erhöhten Wertungsmaßes hier häufig vorkommen kann, und zum anderen wird die Frageperspektive für die Entscheidung festgelegt, indem die Behörde nicht mehr offen (bi- oder multipolar) prüfen muss, welche Entscheidung sie nach ihrem Ermessen für richtig hält, sondern unipolar nur noch prüft, ob ein Ausnahmefall eine andere als die intendierte Entscheidung rechtfertigt. Das sind exakt die Funktionen einer Wertungslastregel, die folglich im „intendierten Ermessen“ stets enthalten ist.

\section{e) Die strafrechtlichen Regelbeispiele}

Im Strafrecht finden sich Wertungslastregeln ausdrücklich in den sog. Regelbeispielen für „besonders schwere Fälle“. Diese formulieren typischerweise „Ein besonders schwerer Fall liegt in der Regel vor, wenn der Täter ... “, gefolgt von Tatbestands-

112 So etwa BVerwGE 72, 1 (6); 91, 82 (90); zu Recht krit. Detterbeck, Allgemeines Verwaltungsrecht (Fn. 110), Rn. 322.

113 Vgl. BVerwGE 105, 55 (57f.); BayVGH NVwZ-RR 2003, 88 (89); VGH Baden-Württemberg VBlBW 2006, 354 (357). 
merkmalen, die in der Regel für eine Qualifikation sorgen sollen (vgl. z. B. $\mathbb{S} 243$ Abs. 1 S. 2, 253 Abs. 4 S. 2, 263 Abs. 3 S. 2 StGB). ${ }^{114}$ Wie bei $\mathbb{} 307$ Abs. 2 BGB auch $^{115}$ geht es um die Konkretisierung der generellen Abwägungsentscheidung, die zu treffen ist, um zu ermitteln, ob ein bestimmter Sachverhalt einen besonders schweren Fall darstellt oder nicht.

Bei der Prüfung, ob ein besonders schwerer Fall vorliegt, hat das Gericht normalerweise anhand einer umfassenden Abwägung aller Umstände des Einzelfalles zu ermitteln, ob der Sachverhalt von den „normalen“ Fällen eines Diebstahls, einer Erpressung oder eines Betrugs derart abweicht, dass die Anwendung des höheren Strafrahmens geboten erscheint. ${ }^{116}$ Liegen allerdings die tatsächlichen Voraussetzungen eines Regelbeispiels vor, so wird vermutet, dass es sich um einen besonders schweren Fall handelt; das Gericht muss aber gleichwohl unter Abwägung aller Umstände des Einzelfalls prüfen, ob nicht doch ausnahmsweise die Anwendung des höheren Strafrahmens nicht gerechtfertigt ist. ${ }^{117}$ Inhaltlich ist sowohl bei den „unbenannten “ besonders schweren Fällen als auch bei den Regelbeispielen jeweils eine strafzumessungsrechtliche Abwägungsentscheidung unter Würdigung aller Umstände des Einzelfalles zu treffen, ob die Umstände die Anwendung des höheren Strafrahmens erfordern oder nicht. ${ }^{118}$ Der einzige Unterschied, den die Erfüllung eines Regelbeispiels bewirkt, ist die Reduktion der Frageperspektive und die Umkehr der Wertungslast: Gelangt das Gericht bei Vorliegen eines Regelbeispiels nicht zu einer abweichenden Überzeugung, so bleibt es bei der Anwendung des verschärften Strafrahmens. ${ }^{119}$

\section{Die Verteilung der Wertungslast}

Den vorstehend genannten Beispielen für gesetzliche Wertungslastregeln lässt sich bereits eine wesentliche Aussage über die Verteilung der Wertungslast entnehmen: Sie wird vom Gesetzgeber implizit durch die Formulierung der Normen mitgeregelt. ${ }^{120}$ Insoweit unterscheidet sie sich nicht von der Verteilung der Beweislast, für die nach heute ganz herrschender Auffassung ebenfalls im Ausgangspunkt die sog.

114 Vgl. dazu eingehend J. Eisele, Die Regelbeispielsmethode im Strafrecht, Tübingen 2004, S. 18 ff.; weitere Beispiele auch bei M. Hettinger, Die Strafrahmen des StGB nach dem Sechsten Strafrechtsreformgesetz, in: M. Hettinger/J. Zopfs u. a. (Hrsg.), FS Küper, Heidelberg 2007, S. 95 (105 ff.).

115 Vgl. dazu soeben C.IV.3.b) a. E.

116 Vgl. BGH NJW 1990, 1489 (1489f.); W. Stree/J. Kinzig, in: A. Schönke/H. Schröder (Hrsg.), Strafgesetzbuch, 28. Aufl., München 2010, Vor $\$ \$ 38$ ff. Rn. 50.

117 Vgl. BGHSt 23, 257; 24, 248 (249); BGH NStZ 2004, 265 (266); s. zum Ganzen bereits J. Wessels, Zur Problematik der Regelbeispiele für „schwere“ und „besonders schwere“ Fälle, in: F.-C. Schröder (Hrsg.), FS Maurach, Karlsruhe 1972, S. 295 (301 ff.); Eisele, Regelbeispielsmethode (Fn. 114), S. $191 \mathrm{ff}$.

118 Vgl. Eisele, Regelbeispielsmethode (Fn. 114), S. 206, der allerdings selbst eine abweichende Auffassung vertritt, vgl. ebd. S. 209.

119 Vgl. nur die Nachweise bei Eisele, Regelbeispielsmethode (Fn. 114), S. 197 ff.

120 Vgl. zu diesem grundsätzlichen Gleichlauf von Beweis- und Wertungslast Riehm, Abwägungsentscheidungen (Fn. 9), S. 139 f. 
Normentheorie gilt. ${ }^{121}$ Hier wie dort obliegt es in erster Linie dem Gesetzgeber, das normative Regel-Ausnahme-Verhältnis festzulegen, das mit Beweislast- und Wertungslastregeln verbunden ist. Indem der Gesetzgeber Anspruchsvoraussetzungen als anspruchsbegründende Tatbestandsmerkmale formuliert, bestimmt er, dass der $A n$ spruchsteller hierfür die Beweis- und Wertungslast trägt; durch ihre Formulierung als anspruchshindernde bzw. anspruchsvernichtende Tatbestandsmerkmale überträgt er die Beweis- und Wertungslast dem Anspruchsgegner. ${ }^{122}$ Der Rechtsanwender muss daher meist keine besonderen Erwägungen anstellen, um die Verteilung der Beweis- und Wertungslast zu ermitteln: Solange er sich an die Maxime „Jede Partei trägt die Beweis- und Wertungslast für diejenigen Tatbestandsmerkmale, auf die sie sich beruft" hält, ergibt sich die richtige Verteilung in aller Regel von selbst aufgrund der hierauf abgestimmten Gesetzesfassung.

Dass sich der Gesetzgeber auch für die Verteilung der Wertungslast an der Normentheorie orientiert, wird etwa an der vermeintlich umständlichen doppelten Verneinung in $\$ 1$ Abs. 2 HGB deutlich. ${ }^{123}$ Rein sprachlich betrachtet wäre die folgende Fassung der Norm bedeutungsgleich, die auf die unschöne doppelte Verneinung verzichtet: „Handelsgewerbe ist jeder Gewerbebetrieb, dessen Unternehmen nach Art und Umfang einen in kaufmännischer Weise eingerichteten Geschäftsbetrieb erfordert." Bei dieser Formulierung würde aber die durch $\$ 1$ Abs. 2 HGB bewirkte Verteilung der (Beweis- und) Wertungslast umgekehrt, denn nunmehr müsste derjenige die Wertungslast tragen, der sich auf die Kaufmannseigenschaft beruft, anstatt desjenigen, der ihr Fehlen behauptet.

\section{Kontrollmaßstäbe für Einzelfallabwägungen}

Auf Grundlage der vorstehend entwickelten Struktur der Einzelfallabwägung können auch die Kriterien für die Richtigkeit, mithin die Maßstäbe einer Falsifikation, ermittelt werden.

\section{Gegenstand der Kontrolle}

Vorab ist zu erörtern, worauf sich die Kontrolle von Einzelfallabwägungen bezieht: Denkbare Kontrollgegenstände sind insoweit der innere psychologische Entscheidungsvorgang, die nach außen gegebene Entscheidungsbegründung oder das Ent-

121 Vgl. grundlegend Rosenberg, Beweislast (Fn. 80), S. 116, 126 ff.; aus neuerer Zeit etwa Musielak, Grundlagen der Beweislast (Fn. 88), S. 292 ff.; Prütting, Gegenwartsprobleme (Fn. 76), S. 265 ff., 277; differenzierend Leipold, Beweismaß und Beweislast (Fn. 72), S. 17 ff.; ders. (Fn. 80), $\$ 286$ Rn. $69 \mathrm{f}$.

122 Vgl. eingehend Riehm, Abwägungsentscheidungen (Fn. 9), S. 136 ff.

123 Vgl. für zahlreiche weitere Beispiele Riehm, Abwägungsentscheidungen (Fn. 9), S. 140 ff. 
scheidungsergebnis. ${ }^{124}$ Der innere Entscheidungsvorgang scheidet dabei schon deswegen als Kontrollgegenstand aus, weil er gerade nicht nach außen dringt und daher schon faktisch einer Kontrolle entzogen ist. Ob und auf welche Weise und aufgrund welcher Erwägungen das zur Entscheidung berufene Organ tatsächlich seine Entscheidung getroffen hat, bleibt sein Geheimnis. Nach außen dringt allein die gegebene Entscheidungsbegründung, für die der Entscheider die Verantwortung übernimmt. Dass diese möglicherweise eine Scheinbegründung ist, ${ }^{125}$ also von den tatsächlichen Motiven des Entscheiders abweicht, mag verwerflich sein und kann im Entdeckungsfall die Entscheidung auch disqualifizieren, ${ }^{126}$ macht die wahren Gründe aber per definitionem nicht erkennbar und daher auch nicht kontrollfähig.

Damit bleiben nur die verlautbarte Entscheidungsbegründung und das Entscheidungsergebnis als mögliche Kontrollgegenstände. Eine Kontrolle des Entscheidungsergebnisses setzt allerdings voraus, dass eine Abwägungsentscheidung stets zu dem „einen richtigen“ Ergebnis führt, wodurch jedes andere Ergebnis falsifiziert würde; dies würde eine „Verifikation“ der Entscheidung bedeuten. Dafür wären allerdings objektive, also intersubjektiv nachvollziehbare Maßstäbe für alle Strukturelemente der Abwägungsentscheidung erforderlich, also auch für die rein einzelfallbezogene konkrete Gewichtung und Gegenüberstellung der Abwägungsgesichtspunkte. Bereits oben wurde ausgeführt, dass derartige Maßstäbe nicht existieren; ${ }^{127}$ der Entscheider ist insoweit darauf angewiesen, seine persönliche Überzeugung zu bilden. Damit scheidet aber auch eine objektive Kontrolle des Abwägungsergebnisses durch einen Außenstehenden aus. Allenfalls könnte ein Außenstehender seine eigene Überzeugung an die Stelle der Überzeugung des Erstentscheiders setzen; einen höheren Anspruch auf objektive Richtigkeit könnte eine solche „Zweitentscheidung“ aber nicht erheben. Lediglich hierarchische oder autoritative Argumente können dazu führen, die Zweitentscheidung für maßgeblich zu erachten, etwa weil sie von einem höherrangigen Gericht oder einem sonst letztentscheidungszuständigen Organ getroffen wurde, dessen Überzeugung das Gesetz für maßgeblich erklärt. ${ }^{128}$

124 S. dazu aus neuerer Zeit etwa K. Gräfin von Schlieffen, Subsumtion als Darstellung der Herstellung juristischer Urteile, in: Gabriel/Gröschner (Hrsg.), Subsumtion (Fn. 24), S. 379 ff.; M. Novak, The Argument from Psychological Typology for a Mild Separation Between the Context of Discovery and the Context of Justification, in: C. Dahlman/E. T. Feteris (Hrsg.), Legal Argumentation Theory, Dordrecht 2013, S. 145 ff.

125 Vgl. zum Problem der Scheinbegründung etwa Engisch, Wahrheit und Richtigkeit (Fn. 71), S. 11 f.; Koch/Rüßmann, Juristische Begründungslehre (Fn.9), S. 115 ff.; Michael, Gleichheitssatz (Fn. 47), S. 64; K. Langenbucher, Das Dezisionismusargument in der deutschen und in der US-amerikanischen Rechtstheorie, ARSP 88 (2002), S. 398 (406); U. Kischel, Die Begründung, Tübingen 2003, S. 10 ff.; C. Bäcker, Begründen und Entscheiden, 2. Aufl., Baden-Baden 2012, S. 34 ff.

126 Vgl. U. Neumann, Juristische Argumentationslehre, Darmstadt 1986, S. 5.

127 Vgl. oben C.II.2 und C.III.

$128 \mathrm{Zu}$ den maßgeblichen Kriterien und Beispielen für die Verteilung von Letztentscheidungskompetenzen vgl. näher Riehm, Abwägungsentscheidungen (Fn. 9), S. $211 \mathrm{ff.}$ 
Zwar sind in der Literatur immer wieder Möglichkeiten behauptet worden, die Richtigkeit von Abwägungsentscheidungen objektiv zu bestimmen. Meistens wird hierbei auf die Diskurstheorie zurückgegriffen und nach einer Form von Konsens gesucht (z. B. empirischer Konsens aller Betroffenen, ${ }^{129}$ Konsens aller vernünftig Denkenden, ${ }^{130}$ Konsens der herrschenden Meinung ${ }^{131}$ ). ${ }^{132}$ Diese Methoden scheitern jedoch für Einzelfallabwägungen schon daran, dass über diese ein wissenschaftlicher Diskurs nicht stattfindet, so dass sich kein Konsens herausbilden kann - unabhängig davon, dass dessen empirische Feststellung in einem realen Gerichtsverfahren ohnehin nicht möglich wäre. ${ }^{133}$ Auch eine Bindung an Präjudizien kommt für Einzelfallabwägungen wegen der Unwiederholbarkeit des konkreten Sachverhalts nicht in Betracht; allenfalls könnte eine Orientierung an Präjudizien erwogen werden, mit denen der konkret zu entscheidende Sachverhalt verglichen wird. Allerdings würde dadurch der Abwägungsspielraum in aller Regel lediglich verkleinert, nicht aber eliminiert, denn auch in diesen Fällen bleibt ein wertender Vergleich des zu entscheidenden Sachverhalts mit dem schon entschiedenen Sachverhalt erforderlich, bei dem sich strukturell die gleichen Probleme stellen.

Ist damit das Entscheidungsergebnis einer Einzelfallabwägung kein tauglicher Gegenstand einer objektiven Richtigkeitskontrolle, so bleibt nur die Möglichkeit einer Kontrolle des Entscheidungsverfahrens, d. h. der nach außen verlautbarten Entscheidungsbegründung. Es verwundert daher nicht, dass Abwägungsentscheidungen in besonderem Maße einem Begründungszwang unterliegen: ${ }^{134} \mathrm{Je}$ weniger die Entscheidung durch das Gesetz determiniert ist, desto größer wird die Bedeutung der Entscheidungsbegründung, d.h. der Wiedergabe der Gründe, aufgrund derer der Entscheider zu seiner Überzeugung gelangt ist. ${ }^{135}$ Dazu zählen bei Abwägungsent-

129 In diese Richtung etwa C. Parchmann, Pluralistische Wirklichkeit und Verwaltungsrecht, München 2003, S. 101 ff. zum Begriff der „Verunstaltung“ im Baurecht; s. zum Problem des empirischen Konsenses auch die Darstellung bei J.-R. Sieckmann, Richtigkeit und Objektivität im Prinzipienmodell, ARSP 83 (1997), S. 14 (30 ff. m. w. N.).

130 So etwa Sieckmann, Richtigkeit und Objektivität im Prinzipienmodell (Fn. 129), S. 24 f.; Kaufmann, Das Verfahren der Rechtsgewinnung (Fn. 34), S. 19 ff., 35 f.; ähnlich in der Sache Alexy, Die Abwägung in der Rechtsanwendung (Fn.14), S. 73 f.; Alexy, Die Gewichtsformel (Fn. 50), S. 773, dort jeweils verstanden als Anforderung an die (material verstandene) Rationalität von Entscheidungen; vgl. dazu noch unten E.I.

131 In diese Richtung etwa Neuner, Die Rechtsfindung contra legem (Fn. 85), S. 73 ff. („herrschende Gerechtigkeitsvorstellungen innerhalb der Sozietät“); s. zum Problem der „herrschenden Meinung“ eingehend T. Drosdeck, Die herrschende Meinung - Autorität als Rechtsquelle, Berlin 1989, S. 107 ff.

132 Auch Viehweg sucht die Lösung für die Zulässigkeit von Argumentationstopoi im Konsens (vgl. T. Viehweg, Topik und Jurisprudenz, 5. Aufl., München 1974, S. 77 ff., v. a. S. 96; s. dazu Bydlinski, Methodenlehre (Fn. 34), S. 143 f.).

133 Vgl. dazu näher Riehm, Abwägungsentscheidungen (Fn. 9), S. 167 ff.

134 Vgl. z. B. $\$ 39$ Abs. 1 S. 3 VwVfG für die Begründung von Ermessensentscheidungen oder $\$ 286$ Abs. 1 S. 2 ZPO für die Begründung der Beweiswürdigung.

135 Vgl. nur S. Schwab, Die Begründungspflicht nach $\$ 39$ VwVfG, Pfaffenweiler 1991, S. 17 ff.; Kischel, Die Begründung (Fn. 125), S. 41 f.; W. Gast, Juristische Rhetorik, 4. Aufl., Heidelberg 2006, Rn. 39 f.; Neumann, Recht als Struktur und Argumentation (Fn. 29), S. 76 ff. 
scheidungen die Konkretisierung des Abwägungsprogramms, die Erläuterung der Abwägungsgesichtspunkte, sowie eine nachvollziehbare Abwägung i.e.S. ${ }^{136}$ Die Entscheidungsbegründung kann dann jedenfalls im Hinblick auf die Einhaltung der prozeduralen Anforderungen an Abwägungsentscheidungen überprüft und ggfs. falsifiziert werden.

Diese Kontrolle schließt zwar nicht aus, dass es unterschiedliche Entscheidungsergebnisse geben kann, die jeweils aufgrund einer prozedural nicht falsifizierbaren Einzelfallabwägung getroffen wurden, also „vertretbar“ sind; eine Verifikation des „einen richtigen“ Entscheidungsergebnisses scheidet daher aus. Gleichwohl sorgt aber die prozedurale Falsifikationsmöglichkeit für eine größtmögliche Richtigkeitsgewähr; die verbleibenden Abweichungsmöglichkeiten der Tatgerichte im Rahmen der vertretbaren Entscheidungen sind im Hinblick auf die offene Natur der verwendeten Tatbestandsmerkmale unvermeidbar. Solange jedoch „nur“ Einzelfallentscheidungen betroffen sind, die nach der hier herausgearbeiteten Definition gerade keine Bedeutung über den Einzelfall hinaus entfalten, ist damit keine unzumutbare Rechtsunsicherheit betroffen. ${ }^{137}$

\section{Prozedurale Abwägungsregeln}

Die prozeduralen Abwägungsregeln, auf deren Einhaltung Einzelfallabwägungen kontrolliert werden können, sollen hier in absolut-objektive Regeln und relativ-objektive Regeln unterteilt werden. Während die absolut-objektiven Regeln unabhängig von der Person des Rechtsanwenders feststehen, also nicht ihrerseits durch eine Abwägung im Rahmen der Obersatzbildung (Auslegung oder Rechtsfortbildung) gewonnen werden, sind die relativ-objektiven Regeln das Ergebnis der Rechtsanwendung, nämlich der Auslegung der anzuwendenden Norm. Diese Auslegung ist ihrerseits nicht vollständig determiniert, sondern unterliegt - auf der Ebene der Obersatzbildung - wiederum den gleichen Defiziten objektiver Richtigkeitskontrolle. Auch hier kann ein letztverbindliches Auslegungsergebnis nicht verifiziert, sondern nur durch Verweis auf hierarchische bzw. autoritative Gesichtspunkte gerechtfertigt werden, insbesondere durch die praktische Verbindlichkeit der Auslegungsergebnisse der jeweiligen Revisionsgerichte, denen die Kompetenz verliehen ist, von ihnen nicht geteilte Auslegungen als „rechtsfehlerhaft“ ${ }^{\text {“ }}$ zu verwerfen. ${ }^{138}$

136 Vgl. BVerfGE 115, 205 (246 ff.).

137 Vgl. näher Riehm, Abwägungsentscheidungen (Fn. 9), S. 193 ff.

138 Dazu näher Riehm, Abwägungsentscheidungen (Fn. 9), S. 211 ff. 
Die absolut-objektiven Maßstäbe beschreiben Grundanforderungen an die formale Rationalität rechtlicher Entscheidungen: ${ }^{139}$ Jede rechtliche Entscheidung - auch die Würdigung aller Umstände des Einzelfalles - muss zunächst in sich widerspruchsfrei sein, d. h. die Argumentation muss in sich konsistent sein, und darf nicht auf sachfremden Erwägungen beruhen. Als weiterer absolut-objektiver Maßstab kommt hin$\mathrm{zu}$, dass die Entscheidung überhaupt in der Form einer Abwägung stattgefunden haben muss, nicht etwa zufällig oder willkürlich.

$\mathrm{Zu}$ den relativ-objektiven Regeln zählen zunächst die positiven und negativen Vorgaben über die zu berücksichtigenden Abwägungsgesichtspunkte: Welche Aspekte müssen, welche dürfen und welche dürfen nicht berücksichtigt werden? ${ }^{140}$ Hinzu kommen die Vorgaben hinsichtlich des abstrakten Gewichts der Aspekte ihrer Art nach, das meist relativ zum abstrakten Gewicht anderer Aspekte vorgegeben wird. ${ }^{141}$ Weitere abstrakt-generelle Vorgaben betreffen das Wertungsmaß - also die Frage, in welchem Maße die Aspekte für die eine Seite die Aspekte für die andere Seite überwiegen müssen - sowie die Wertungslast, also die Frage, wer das Risiko einer fehlenden Überzeugung des Gerichts trägt.

Diese relativ-objektiven Maßstäbe sind - anders als die absolut-objektiven Maßstäbe - nicht jeder rationalen Rechtsanwendung (absolut) vorgegeben. Vielmehr sind sie relativ in dem Sinne, dass ihre Herleitung selbst nicht streng deterministisch erfolgt, sondern von subjektiven Richtigkeitsüberzeugungen im Rahmen der Konkretisierung des Obersatzes durch Auslegung und Rechtsfortbildung abhängt. Dadurch findet über sie ein wissenschaftlicher Diskurs statt, bei dem unterschiedliche Meinungen (etwa zur Verteilung der Wertungslast oder zu den berücksichtigungsfähigen Kriterien $)^{142}$ vertretbar sind, ohne dass eine von ihnen objektiv falsifizierbar wäre. Dieser Diskurs ist Teil des eigentlichen rechtswissenschaftlichen Diskurses, der zwar prinzipiell unendlich ist, aber durchaus auch zu Konsensen führen kann, die zumindest temporär Bestand haben. Jedenfalls aber besteht für diesen Diskurs die Autorität der jeweils obersten Gerichtsinstanzen, die für die Praxis befugt sind zu definieren, welche Maßstäbe als richtig gelten.

139 Der ebenfalls absolut-objektive Maßstab der empirischen Richtigkeit (vgl. Riehm, Abwägungsentscheidungen [Fn. 9], S. 180 f.) spielt bei der Einzelfallwürdigung keine Rolle, weil die verwendeten Abwägungsgesichtspunkte hier keine empirischen Tatsachen der Lebenswelt sind, sondern lediglich die in verfahrensordnungsgemäßer Weise festgestellten Tatsachen, die - wegen Beweisproblemen oder Beweislastentscheidungen - ohne Rechtsfehler von den tatsächlichen Begebenheiten abweichen können.

140 Vgl. oben C.I.

141 Vgl. oben C.II.1.

142 So ist etwa zu $\$ 275$ Abs. 2 BGB umstritten, ob als Bestandteil des Leistungsinteresses des Gläubigers auch immaterielle Interessen zu berücksichtigen sind, vgl. verneinend etwa H. Ehmann/H. Sutschet, Modernisiertes Schuldrecht, München 2002, S. 53 f.; bejahend Finn, Erfüllungspflicht und Leistungshindernis (Fn. 66), S. 296 f.; M. Löwisch, in: Staudinger (Hrsg.), BGB, Berlin 2009, $\$ 275$ Rn. 92 ; Ernst (Fn. 52), $\$ 275$ Rn. 80. 
Die genannten Entscheidungsmaßstäbe sind aber objektiv in dem Sinne, dass - akzeptiert man ihre Geltung aufgrund eigener Überzeugung oder höherer Autorität eine objektive Falsifikation einer Einzelfallentscheidung anhand ihrer möglich ist, wenn etwa das Gericht eine abweichende Wertungslastverteilung zu Grunde gelegt oder im Rahmen der Einzelfallwürdigung auf unzulässige Gesichtspunkte abgestellt hat. Weil diese relativ-objektiven Kontrollmaßstäbe der konkreten Einzelfallabwägung in abstrakt-genereller Weise vorgegeben sind, sind sie im Rahmen des Justizsyllogismus dem Obersatz zuzurechnen; sie können daher auch als abwägungslenkende Obersätze bezeichnet werden. ${ }^{143}$

\section{Verbleibende nicht falsifizierbare Elemente der Abwägung}

Objektive Falsifikationsmaßstäbe können nur für diejenigen Elemente der Abwägung aufgestellt werden, die tatsächlich verallgemeinerbar sind. Die letzten Schritte der eigentlichen Abwägung, also die konkrete Gewichtung der Umstände im Einzelfall und die abschließende Gegenüberstellung der gewichteten Aspekte zur Ermittlung des Abwägungsergebnisses sind dagegen nicht mehr generalisierbar. Vielmehr ist das Gericht, wie bereits oben ausgeführt, insoweit allein auf seine subjektive Überzeugung angewiesen - wie bei der Beweiswürdigung auch. Eine kontrollierende Instanz kann zwar eine eigene, evtl. abweichende Gewichtung und Abwägung der Umstände vornehmen. Diese Abwägung könnte aber keinen höheren Anspruch auf objektive inhaltliche Richtigkeit erheben, so dass es sich hier nicht mehr um eine (objektive) Richtigkeitskontrolle handelt, sondern um eine (subjektive) Zweitentscheidung. Welche der beiden subjektiven Entscheidungen von Rechts wegen als richtig gilt, ist keine Frage objektiver Regeln mehr, sondern kann für die Praxis nur durch Zuweisung von Letztentscheidungskompetenzen geklärt werden. ${ }^{144}$ Hier handelt es sich mithin um den residualen, nicht weiter objektiv überprüfbaren Bereich richterlicher Entscheidung, der allein der Verantwortung und Überzeugung des einzelnen Richters bzw. Spruchkörpers übertragen ist.

Darin liegt kein Verweis auf richterliche Willkür ${ }^{145}$ und auch keine Beschränkung des rechtsstaatlichen Anspruchs an die Entscheidung, ${ }^{146}$ sondern lediglich ein Eingeständnis der Unmöglichkeit, ein deterministisches Gesamtsystem aufzustellen, welches das Ergebnis von Abwägungsentscheidungen abschließend vorgibt und vom Gericht nur noch angewendet werden muss. Dass der Richter nicht durch einen „Subsumtionsautomaten“ ersetzt werden kann, der für jeden Rechtsfall eine eindeu-

143 Vgl. näher Riehm, Abwägungsentscheidungen (Fn. 9), S. 30 ff.

144 Vgl. zu diesem pragmatischen Richtigkeitsbegriff eingehend Riehm, Abwägungsentscheidungen (Fn. 9), S. 173 ff. sowie bereits oben D.I.

145 Dies befürchtet Freund, „Rechts“-Folgenlegitimation (Fn. 16), S. 996 f.

146 Dies befürchten die Vertreter einer fundamentalkritischen Haltung zur Abwägung, etwa B. Schlink, Abwägung im Verfassungsrecht, Berlin 1976; Leisner, Der Abwägungsstaat (Fn. 15); s. hiergegen zutreffend Osterkamp, Juristische Gerechtigkeit (Fn. 71), S. 237. 
tige Lösung aus dem Gesetz ableitet, ist gesicherter Bestand der heutigen Methodenlehre. ${ }^{147}$ Vielmehr dient die vorliegend entwickelte Abwägungsstruktur gerade dazu, den Raum richterlicher Eigenwertung zu beschränken auf diejenigen Teile der Abwägungsentscheidung, in denen sie unumgänglich ist, und die Entscheidung im Übrigen auf kontrollierbare (Verfahrens-)Regeln zurückzuführen. ${ }^{148}$

\section{E. Rechtstheoretische Folgerungen}

\section{Einzelfallabwägung und Rationalität rechtlicher Entscheidungen}

Abwägungsentscheidungen stehen offenbar stets unter dem Generalverdacht, keine rationalen Entscheidungen, ${ }^{149}$ ja sogar rechtsstaatswidrig zu sein. ${ }^{150}$ Dieser Vorwurf wiegt schwer, und es ist viel Energie darauf verwendet worden, ihn zu widerlegen. ${ }^{151}$ Diese umfassende Diskussion kann und muss hier nicht nachvollzogen werden, weil sie sich in weiten Teilen mit dem Gegensatz zwischen Gesetzesbindung und (vermeintlich) „freier“ Abwägung im Bereich der verfassungsrechtlichen Güterabwägung befasst, ${ }^{152} \mathrm{der}$ im Bereich der Würdigung aller Umstände des Einzelfalles keine Bedeutung erlangt, weil es hier das Gesetz selbst ist, das durch die Verwendung unbestimmter Rechtsbegriffe bzw. normativer Tatbestandsmerkmale auf eine richterliche Abwägungsentscheidung verweist. Im Übrigen entpuppt sich die Debatte um die - gegebene oder nicht gegebene - Rationalität von Abwägungsentscheidungen ganz überwiegend als Debatte über den an die Rechtsanwendung anzulegenden Rationalitätsbegriff. ${ }^{153}$

Insoweit liegt eine erste und entscheidende Weichenstellung in der von Max Weber begründeten Unterscheidung zwischen formaler und materialer Rationalität. ${ }^{154}$ Die formale Rationalität (auch: Zweckrationalität) beschreibt Anforderungen an das Verfahren der Entscheidungsfindung bzw. -begründung, die materiale Rationalität

147 Vgl. nur N. Hoerster, Verteidigung des Rechtspositivismus, Frankfurt a. M. 1989, S. 12 f.; Osterkamp, Juristische Gerechtigkeit (Fn. 71), S. 58 f.; s. hierzu im historischen Kontext eingehend $R$. Ogorek, Richterkönig oder Subsumtionsautomat?, 2. Aufl., Frankfurt a. M. 2008.

148 Vgl. in diesem Sinne auch Canaris, Systemdenken (Fn. 36), S. 144 f., 148 f.; ders., Richtigkeit und Eigenwertung (Fn. 80), S. 40 f.

149 Vgl. etwa G. Struck, Interessenabwägung als Methode, in: R. Dubischar (Hrsg.), Dogmatik und Methode - Josef Esser zum 65. Geburtstag, Kronberg/Ts. 1975, S. 171 (172 ff., 176 ff.); Schlink, Abwägung im Verfassungsrecht (Fn. 146), S. 127 ff.; ders., Der Grundsatz der Verhältnismäßigkeit, in: P. Badura/H. Dreier (Hrsg.), FS BVerfG, Bd. 2, Tübingen 2001, S. 445 (460 ff.); J. Habermas, Faktizität und Geltung, 4. Aufl., Frankfurt a. M. 1994, S. 315 f.

150 Deutlich etwa bei Leisner, „Abwägung überall“ (Fn. 15).

151 Vgl. etwa Hubmann, Wertung und Abwägung (Fn. 18), S. 1 ff., 145 ff.; J.-R. Sieckmann, Zur Begründung von Abwägungsurteilen, RTh 26 (1995), S. 45 ff.; N. Jansen, Die Struktur rationaler Abwägungen, ARSP-Beiheft 66 (1997), S. 152 ff.; Alexy, Die Gewichtsformel (Fn. 50), S. 771 ff.

152 So insbesondere Leisner, „Abwägung überall“ (Fn. 15); Leisner, Der Abwägungsstaat (Fn. 15) und Schlink, Abwägung im Verfassungsrecht (Fn. 146); Schlink, Der Grundsatz der Verhältnismäßigkeit (Fn. 149), S. $445 \mathrm{ff}$.

153 Vgl. dazu eingehend Riehm, Abwägungsentscheidungen (Fn. 9), S. 94 ff.

154 Vgl. M. Weber, Wirtschaft und Gesellschaft, 3. Aufl., Tübingen 1947, S. 44 f. 
(auch: Wertrationalität) geht darüber hinaus und beschreibt auch Anforderungen an das Entscheidungsergebnis. Der Idealfall der formalen Rationalität ist dabei die deterministische Berechenbarkeit des Entscheidungsergebnisses aus den Prämissen nach Art einer mathematischen Formel. ${ }^{155}$ Die materiale Rationalität verlangt darüber hinaus die „inhaltliche Richtigkeit“ der Entscheidung - ihre Grenzen wurden bereits im Rahmen der Kontrollmöglichkeiten für Einzelfallabwägungen diskutiert, mit dem Ergebnis, dass eine materiale Rationalität in diesem Sinne tatsächlich durch Einzelfallabwägungen nicht gewährleistet werden kann, weil es an objektiven inhaltlichen Maßstäben für eine Ergebniskontrolle fehlt. ${ }^{156}$

Was die formale Rationalität der Entscheidung anbelangt, so ist das Weber'sche Ideal der prozeduralen Determination des Entscheidungsergebnisses im Sinne einer zwingend von jedem fehlerfreien Rechtsanwender zu erzielenden Lösung in der Tat nicht erreichbar, weil für die konkrete Gewichtung und die Abwägung selbst in aller Regel intersubjektive Maßstäbe fehlen. Dieses Ideal ist in der Rechtsanwendung allerdings allenfalls bei Subsumtionsschlüssen innerhalb eines geschlossenen axiomatischen Begriffssystems erreichbar, also etwa bei der Unterordnung der Übereignung i. S. v. \929 S. 1 BGB unter den Begriff der Verfügung i. S. v. $\$ 185$ Abs. 1 BGB oder $\mathbb{} 816$ Abs. 1 BGB. Im Regelfall erfordert die Rechtsanwendung indessen Wertungen des Rechtsanwenders, die notwendig subjektiv sind und daher trotz Einhaltung aller prozeduralen Regeln im Ergebnis abweichen können. ${ }^{157}$ Wer also Rationalität mit Determination des Ergebnisses gleichsetzt, muss konsequenterweise jede Rechtsanwendung als irrational bezeichnen.

Allerdings ist die Determination des Ergebnisses nicht die einzige denkbare Auffassung von formaler Rationalität. So wird in der Psychologie Rationalität etwa als Freiheit von Emotionen verstanden; rationale Entscheidungen sind solche, die nicht durch Emotionen, Triebe, Instinkte und spontane Impulse beeinflusst sind. ${ }^{158}$ Dieser Rationalitätsbegriff wäre zwar als praktische Anforderungen an rechtliche Entscheidungen nicht durchsetzbar, weil die tatsächliche Motivationslage des Entscheiders nicht nach außen dringt und daher nicht kontrolliert werden kann. Sie zeigt aber, dass das Weber'sche Ideal der formalen Rationalität durch Determination der Entscheidung nicht die einzig denkbare Lesart des Rationalitätsbegriffes ist. Erst recht verlangt das Rechtsstaatsprinzip nicht die vollständige Determination des Entscheidungsergebnisses. Dieses verlangt nämlich lediglich, dass das Entscheidungsergebnis

155 Vgl. Weber, Wirtschaft und Gesellschaft (Fn. 154), S. 45.

156 Vgl. oben D.

157 Vgl. nur R. Alexy, Theorie der Grundrechte, Frankfurt a. M. 1994, S. 149; Larenz/Canaris, Methodenlehre (Fn. 28), S. 223 mit Fn. 93; Michael, Gleichheitssatz (Fn. 47), S. 300 f.; Sieckmann, Richtigkeit und Objektivität (Fn. 129), S. 29; M. Auer, Materialisierung, Flexibilisierung, Richterfreiheit, Tübingen 2005, S. 133; Röhl/Röhl, Allgemeine Rechtslehre (Fn. 36), $\$ 32$ IV 4 a. E. (S. 280 f.).

158 Vgl. die Nachweise bei J. J. Hagen, Rationales Entscheiden, München 1974, S. 63 mit N. 30. 
mit „vernünftigen“ Gründen aus Gesetz und Recht abgeleitet werden kann. ${ }^{159}$ Eine Entscheidung kann danach schon dann als rational bezeichnet werden, wenn ein sachlicher Begründungszusammenhang zwischen den Prämissen (Sachverhalt und Obersatz) und dem Entscheidungsergebnis besteht. ${ }^{160}$ Hierfür ist nicht erforderlich, dass das Entscheidungsergebnis aus den Gründen zwingend folgt; es genügt, wenn es hieraus folgen kann, dass also ein Begründungszusammenhang etabliert werden kann, der die Entscheidung an Gesetz und Recht einerseits und an den zu entscheidenden Sachverhalt andererseits rückbindet. ${ }^{161}$ Dafür müssen alle relevanten - und keine irrelevanten - Gesichtspunkte tatsächlich bei der Abwägungsentscheidung in widerspruchsfreier Weise im Rahmen einer nachvollziehbaren Argumentation berücksichtigt worden sein.

Legt man diesen Rationalitätsbegriff zu Grunde, so ist jede Einzelfallabwägung, die die vorstehend entwickelte Abwägungsstruktur einhält, zugleich eine rationale Entscheidung.

\section{Einzelfallabwägung und „One Right Answer"-These}

In erster Linie auf den jüngst verstorbenen US-amerikanischen Rechtsphilosophen Ronald Dworkin geht die „One Right Answer“-These zurück: ${ }^{162}$ Nach dieser These gibt es für jede Rechtsfrage „eine richtige Antwort“, welche der Entscheidung objektiv und ontologisch vorgegeben sei; die Aufgabe des Gerichts sei es, diese zu erkennen und auszusprechen. Die vorstehend entwickelte Abwägungsstruktur erlaubt es zwar, Abwägungsentscheidungen in weitem Rahmen zu falsifizieren, so dass sichergestellt werden kann, dass die im Wege der rationalen Abwägung gefundene Entscheidung nicht falsch ist. Ob die Entscheidung allerdings die einzig richtige ist, kann sie nicht ermitteln. Vielmehr bleibt es möglich, dass unterschiedliche Entscheider trotz Einhaltung derselben Abwägungsprozedur und Berücksichtigung derselben Abwägungsaspekte zu unterschiedlichen Ergebnissen gelangen, weil sie manchen Aspekten abweichende konkrete Gewichte zusprechen und daher bei der Gesamtabwägung zu unterschiedlichen Überzeugungen gelangen. Zwischen diesen unterschiedlichen Ergebnissen kann das „richtige“ weder prozedural noch material er-

159 Vgl. BVerfGE 4, 1 (7); 34, 269 (287); 42, 64 (74); 57, 39 (42); 89, 132 (141); R. Alexy, Theorie der juristischen Argumentation, 2. Aufl., Frankfurt a. M. 1990, S. 11; Michael, Gleichheitssatz (Fn. 47), S. $66,2235 \mathrm{ff}$.

160 S. dazu eingehend Riehm, Abwägungsentscheidungen (Fn. 9), S. 98 ff.; zustimmend S. Unger, Das Verfassungsprinzip der Demokratie, Tübingen 2008, S. 138; H.-J. Papier/C. Krönke, Grundkurs Öffentliches Recht 2, Heidelberg u.a. 2012, Rn. 155.

161 Vgl. auch C. Bäcker, Der Syllogismus als Grundstruktur des juristischen Begründens?, RTh 40 (2009), S. 404 (409).

162 Vgl. R. Dworkin, Bürgerrechte ernstgenommen, Frankfurt a. M. 1984, S. 448 ff.; ders., A Matter of Principle, Cambridge (Mass.)/London 1985, S. 119 ff.; zu Rezeption und Kritik vgl. etwa Canaris, Richtigkeit und Eigenwertung (Fn. 80); T. Herbst, Die These der einzig richtigen Entscheidung, JZ 2012, S. 891 ff., jeweils m.w. N. 
mittelt werden: Prozedural nicht, weil die Abwägungsprozedur ex praemissione eingehalten wurde, und material nicht, weil für die Ermittlung der inhaltlichen Richtigkeit von Abwägungsentscheidung keine objektiven Maßstäbe unabhängig von der rational gewonnenen Überzeugung der jeweiligen Entscheider bestehen. ${ }^{163}$

Auch konsensorientierte materiale Richtigkeitsmaßstäbe, wie sie etwa in der Diskurstheorie Jürgen Habermas' vorgeschlagen wurden, ${ }^{164}$ sind jedenfalls für die hier behandelte Einzelfallabwägung untauglich, weil sie einen breiten und offenen gesellschaftlichen Diskurs über die Richtigkeit einer Entscheidung voraussetzen. Ein solcher findet aber in der Realität weder über jeden Einzelfall statt, noch könnte ein konkretes Gericht vor Verkündung eines Urteils einen solchen Diskurs anregen oder sein Ergebnis zuverlässig ermitteln. Vielmehr ist es gerade das Wesen jedes Diskurses vor Gericht, dass er kontradiktorisch verläuft und eben nicht zu einem Konsens führt (sonst würden sich die Parteien vergleichen und das Gericht bräuchte kein Urteil zu fällen). Solange eine Partei nicht die Regeln des prozessualen Diskurses verletzt, indem sie unsachlich oder irrational im oben dargelegten Sinne argumentiert, solange also verschiedene Positionen rational begründbar bleiben und von den Parteien des Rechtsstreits beibehalten werden, führt der rationale Diskurs nicht zu einem eindeutigeren Ergebnis als die Abwägung selbst. Für die praktische Rechtsanwendung im konkreten Einzelfall ist das Diskursmodell daher untauglich. ${ }^{165}$

Danach ist weder objektiv-ontologisch im Sinne Dworkins noch objektiv-kommunikativ im Sinne Habermas' die Ermittlung einer „einzig richtigen Entscheidung“ möglich. Allerdings behauptet selbst Dworkin nicht, dass die von ihm postulierte „eine richtige Antwort“ tatsächlich unter den realen Bedingungen praktischer Rechtsfindung zu ermitteln wäre. Hierzu bedürfte es - in den Worten Dworkins eines „Herkules“, eines „Juristen mit übermenschlicher Fertigkeit, Ausbildung, Geduld und Scharfsinn “. ${ }^{166}$ Er bestreitet ausdrücklich, dass ein Entscheidungsprozess in hard cases bei verschiedenen Richtern $\mathrm{zu}$ den gleichen Ergebnissen führen wird. ${ }^{167}$ Die „eine richtige Antwort“ entpuppt sich dann als ideales Ziel der Rechtsanwendung, als „regulatives Prinzip“ im Sinne Kants. ${ }^{168}$ Die Vorstellung von der

163 Vgl. dazu eingehend Riehm, Abwägungsentscheidungen (Fn. 9), S. 166 ff.

164 S. dazu insbesondere Habermas, Faktizität und Geltung. (Fn. 149), S. 272 ff.; zur Kritik vgl. etwa Herbst, Einzig richtige Entscheidung (Fn. 162), S. 897 ff. m. w. N.

165 Auch für abstrakte Rechtsfragen führt es nicht zu eindeutig ermittelbaren Ergebnissen, die ein Richter bei seiner Entscheidung berücksichtigen könnte, insbesondere nicht in den hard cases, vgl. näher Riehm, Abwägungsentscheidungen (Fn. 9), S. $167 \mathrm{ff}$.

166 Dworkin, Bürgerrechte ernstgenommen (Fn. 162), S. 182.

167 Dworkin, Bürgerrechte ernstgenommen (Fn. 162), S. 449; ebenso A. Kaufmann, Das Schuldprinzip, 2. Aufl., Heidelberg 1976, S. 60, 260 f.

168 Vgl. Kant, Kritik der reinen Vernunft (Fn. 84), S. B 537; s. zu dieser Parallele auch Canaris, Richtigkeit und Eigenwertung (Fn. 80), S. 41; R. Alexy, Ota Weinbergers Kritik der diskurstheoretischen Deutung juristischer Rationalität, in: P. Koller/W. Krawietz u. a. (Hrsg.), Institution und Recht, Berlin 1994, S. 143 (149 f.); Lege, Pragmatismus und Jurisprudenz (Fn. 70), S. 413 f.; Osterkamp, Juristische Gerechtigkeit (Fn. 71), S. 73 f.; U. Neumann, Wahrheit im Recht, Baden-Baden 2004, S. 39 ff. 
Möglichkeit einer richtigen Antwort ist Voraussetzung der Überzeugung des Gerichts von der Richtigkeit seiner Entscheidung, welche wiederum Bestandteil des rationalen Entscheidungsvorganges ist. Ohne diese Leitidee hätte das Gericht keine Möglichkeit, unter mehreren rational begründbaren Lösungen diejenige auszuwählen, die es für die richtige ${ }^{169}$ hältt; ${ }^{170}$ geradezu unerträglich und mit dem Wesen des Rechts selbst unvereinbar wäre die Alternative, dass das Gericht seine Entscheidung nicht für richtig hält - der Anspruch auf Richtigkeit ist dem Recht immanent. ${ }^{171}$ Das ändert allerdings nichts daran, dass es keine objektive Möglichkeit gibt, diese Richtigkeitsüberzeugung zu bestätigen oder zu widerlegen.

Realistisch ist lediglich eine pragmatische Vorstellung von der „einen richtigen Antwort" bei einer Einzelfallabwägung: Die von der Rechtsordnung zur Letztentscheidung berufene Instanz - das ist bei Einzelfallabwägungen typischerweise die letzte Tatsacheninstanz ${ }^{172}$ - muss die Abwägungsfrage eindeutig und aufgrund einer rationalen Entscheidungsprozedur entscheiden. Ihre Entscheidung gilt, sofern die prozeduralen Vorgaben eingehalten wurden, für die Praxis insofern als richtig, als sie von keiner anderen Institution in Frage gestellt werden darf. ${ }^{173}$ Näher kann man dem Ideal der einen richtigen Entscheidung in der Rechtspraxis offenbar nicht kommen.

\section{Einzelfallabwägung und Topik}

Die Einzelfallabwägung wird vielfach als (legitimer) Anwendungsfall topischen Denkens in der Jurisprudenz angesehen. ${ }^{174}$ Allerdings ist durchaus unklar, was mit dem Begriff „Topik“ in einem rechtlichen Kontext überhaupt gemeint ist. ${ }^{175}$ Von den

169 Mit Richtigkeit ist hier nicht die Übereinstimmung mit moralischen Überzeugungen gemeint, sondern allein die Richtigkeit nach rechtlichen Maßstäben, so dass der Richter durchaus gezwungen sein kann, gegen sein Gewissen zu entscheiden, wenn er der Überzeugung ist, dass nur diese Entscheidung rechtlich richtig ist; vgl. ebenso Alexy, Theorie der juristischen Argumentation (Fn. 159), S. 351; Bäcker, Begründen und Entscheiden (Fn. 125), S. $215 \mathrm{ff}$.

170 So i. E. auch Sieckmann, Zur Begründung von Abwägungsurteilen (Fn. 151), S. 69; Neumann, Wahrheit im Recht (Fn. 168), S. 39 f.; Herbst, Einzig richtige Entscheidung (Fn. 162), S. 898 f.

171 Vgl. nur Alexy, Theorie der juristischen Argumentation (Fn. 159), S. 351; ders., Begriff und Geltung des Rechts, 2. Aufl., Freiburg/München 1992, S. 64 ff.; Osterkamp, Juristische Gerechtigkeit (Fn. 71), S. $68 \mathrm{f}$.

172 Vgl. näher Riehm, Abwägungsentscheidungen (Fn. 9), S. 233 ff.

173 Vgl. oben Fn. 144.

174 Vgl. U. Diederichsen, Topisches und systematisches Denken in der Jurisprudenz, NJW 1966, S. 697 (704 f.); Canaris, Systemdenken (Fn. 36), S. 76 f.; ders., Bewegliches System und Vertrauensschutz im rechtsgeschäftlichen Verkehr, in: F. Bydlinski/H. Krejci u. a. (Hrsg.), Das Bewegliche System im geltenden und künftigen Recht, Wien u.a. 1986, S. 103 f.; Michael, Gleichheitssatz (Fn. 47), S. 66 f.

175 Vgl. C. Fischer, Topoi verdeckter Rechtsfortbildungen im Zivilrecht, Tübingen 2007, S. 17; grundlegend zur Topik in der Jurisprudenz Viehweg, Topik und Jurisprudenz (Fn. 132); N. Horn, Zur Bedeutung der Topiklehre Theodor Viehwegs für eine einheitliche Theorie des juristischen Denkens, NJW 1967, S. 601 ff.; K. Rehbock, Topik und Recht, München 1988 sowie aus neuerer Zeit A. Launhardt, Topik und rhetorische Rechtstheorie, Frankfurt a. M. 2010, passim; s. aber auch das vernichtende Urteil von O. Weinberger, Überzeugen als Aufgabe, ARSP 81 (1995), S. 305 (308): „Viehwegs Topik-Lehre, die in der deutschen Jurisprudenz eine Zeit lang hoch im Kurs stand, müssen wir hier nicht näher in Betracht ziehen, da sie kaum etwas Wesentliches bringt und heute eigentlich schon tot ist.". 
Befürwortern topischer Argumentationsweise in der Jurisprudenz wird Topik meist verstanden als Denken und Argumentieren ausgehend vom konkreten Problem, d. h. von den spezifischen Tatsachenelementen des Einzelfalls. ${ }^{176}$ Daran trifft für die Einzelfallabwägung zu, dass der Kreis der Abwägungsgesichtspunkte nicht ex ante abschließend feststeht, sondern erst aus dem Material des konkreten Falles entwickelt werden kann. Niemand kann im Vorhinein abschließend sagen, auf welche Aspekte es bei jedem zukünftigen Fall ankommen wird. ${ }^{177}$ Der Obersatz muss mit anderen Worten immer offen sein für die Berücksichtigung neuer, bisher noch nie dagewesener Aspekte, so dass auch kein vollständiges System der berücksichtigungsfähigen Topoi entwickelt werden kann.

Teils wird Topik aber auch als Gegenbegriff zu systematischer, regelgeleiteter Rechtsanwendung verstanden - „Problemdenken“ als Gegenpol zu „Systemdenken“ oder gar zum „syllogistischen Denken“. ${ }^{178}$ Topisches Rechtsdenken solle sich dadurch auszeichnen, dass die hergebrachten juristischen Argumentationskanones ebenso wie der klassische Justizsyllogismus keine Geltung beanspruchten, sondern die Lösung des Falles insgesamt - also nicht nur punktuell - im Wege freien Argumentierens gefunden werde. ${ }^{179}$ In einem solchen Sinne handelt es sich bei der Einzelfallabwägung nicht um topische Rechtsanwendung, weil sie, wie oben gezeigt, in den Justizsyllogismus eingebettet ist. Ihr geht die - regelgeleitete - Bildung eines Obersatzes voraus, und sie steht neben klassischen Subsumtionen unter einfache (deskriptive) Tatbestandsmerkmale. Keinesfalls wird daher der gesamte Fall durch topische Argumentation gelöst; topische Argumentation findet allenfalls in einem klar abgegrenzten Bereich statt, eben zur Subsumtion derjenigen Tatbestandsmerkmale, die auch im konkretisierten Obersatz noch wertausfüllungsbedürftig sind. Aber auch dann hat die topische Argumentation nicht den Charakter „freien Argumentierens“, sondern ist weiter regelgeleitet (und muss das in einem Rechtsstaat auch sein). Die oben so genannten abwägungslenkenden Obersätze sind Regeln, welche die Argumentation strukturieren und insbesondere eine prozedurale Falsifikation des Entscheidungsergebnisses ermöglichen, was bei einer freien topischen Argumentation nicht möglich wäre. Außerdem sind die abwägungslenkenden Obersätze einer gesetzlichen Regelung zugänglich, und selbst wenn eine solche nicht explizit erfolgt,

176 Vgl. etwa Viehweg, Topik und Jurisprudenz (Fn. 132), S. 39, 97.

177 Vgl. etwa Viehweg, Topik und Jurisprudenz (Fn. 132), S. 38 ff.

178 Vgl. Viehweg, Topik und Jurisprudenz (Fn. 132), S. $31 \mathrm{ff} . ;$ in diesem Sinne auch Canaris, Systemdenken (Fn. 36), S. 135 ff.; J. Esser, Grundsatz und Norm in der richterlichen Fortbildung des Privatrechts, 4. Aufl., Tübingen 1990, S. 47, 221 ff.; Bydlinski, Methodenlehre (Fn. 34), S. 142 ff.; s. dazu auch K. I. Lee, Die Struktur der juristischen Entscheidung aus konstruktivistischer Sicht, Tübingen 2010, S. 112 ff.; gegen einen Gegensatz von Topik und Systematik und für die Verschmelzung in einem „topischen System“ O. Ballweg, Phronetik, Semiotik und Rhetorik, in: O. Ballweg/T.-M. Seibert (Hrsg.), Rhetorische Rechtstheorie, Freiburg i. Br. 1982, S. 27 (45 f.).

179 So etwa Bydlinski, Methodenlehre (Fn. 34), S. 142 ff.; Gröschner, Justizsyllogismus? Jurisprudenz! (Fn. 27). 
unterliegt ihre Herleitung durch die Rechtsprechung einerseits der obergerichtlichen Kontrolle und andererseits dem wissenschaftlichen Diskurs über Normen und ihre Auslegung. Über abwägungslenkende Obersätze kann also durchaus diskutiert werden, etwa über die Berücksichtigungsfähigkeit von Aspekten, über ihr abstraktes Gewicht sowie über die Verteilung der Wertungslast. Dieser Diskurs ist nicht topisch im Sinne von „nur“" problemorientiert, sondern im Rahmen der gesetzlichen Wertungen geleitet durch die Regeln der juristischen Auslegungsmethodik - so wie bei jeder Obersatzbildung durch Normauslegung.

Das schließt nicht aus, dass notwendigerweise Elemente im Rechtsanwendungsprozess verbleiben, in denen der Richter tatsächlich problemorientiert argumentieren muss, ohne dass der Gang seiner Argumentation ex ante abschließend vorgegeben ist. Der von manchen geltend gemachte scharfe Gegensatz von System- und Problemdenken besteht aber nicht; ${ }^{180}$ vielmehr haben beide Ansätze ihren Raum in der Rechtsanwendung. Dabei überwiegt das Systemdenken schon aus Gründen der Rechtsanwendungsgleichheit ${ }^{181}$ bei Weitem und reicht genau bis zur Bildung des Obersatzes im hier zu Grunde gelegten Sinne, einschließlich der abwägungslenkenden Obersätze. Nur ,in den letzten Winkeln“, wo die besonderen Umstände des Einzelfalles sich der abstrakt-generellen Formulierung in einer Regel entziehen, ist ein rein problemorientiertes Denken zulässig (und nötig). ${ }^{182}$

\section{F. Zusammenfassung}

1. Die Einzelfallabwägung im hier behandelten Sinne ist die Methode für die Subsumtion eines Sachverhalts unter ein wertausfüllungsbedürftiges Tatbestandsmerkmal im Obersatz. Es handelt sich daher um eine Einzelfallentscheidung, die zu einem Ergebnis führt, nämlich zu der Feststellung, ob ein bestimmtes Tatbestandsmerkmal im konkreten Fall vorliegt oder nicht.

2. Im Rahmen einer solchen Einzelfallabwägung hat das Gericht zunächst das entscheidungsrelevante Material zu sammeln, abstrakt und konkret zu gewichten und einander gegenüber zu stellen, um zu ermitteln, welche Gesichtspunkte überwiegen. Ggfs. ist zu prüfen, ob das Übergewicht der Gesichtspunkte zugunsten einer Seite ein im normativen Tatbestandsmerkmal vorgesehenes erhöhtes Wertungsmaß (z. B. „grobes Missverhältnis“) erreicht. Diese Schritte sind in der Entscheidungsbegründung zu dokumentieren, um eine Kontrolle zu ermöglichen.

3. Gelangt das Gericht am Ende der Abwägung trotz aller Anstrengungen nicht zu einer hinreichenden Überzeugung vom Überwiegen der Abwägungsgesichtspunkte zugunsten einer Seite (non liquet), so hat es seine Entscheidung anhand der Wertungslast zu treffen - obwohl es sich um eine rechtliche Wertungsentschei-

180 Ebenso Bydlinski, Methodenlehre (Fn. 34), S. 143 Fn. 341.

181 Vgl. auch Bydlinski, Methodenlehre (Fn. 34), S. 145 f.

182 Vgl. Viehweg, Topik und Jurisprudenz (Fn. 132), S. 35: „Topik erster Stufe“. 
dung, nicht um eine Tatsachenfeststellung handelt. Die Kategorie der Wertungslast liegt zahlreichen gesetzlichen Regelungen zu Grunde, ist also fester Bestandteil der positiven Rechtsordnung. Ihre Verteilung bestimmt sich wie die der Beweislast nach der sog. Normentheorie.

4. Für Materialauswahl, abstrakte Gewichtung, Wertungsmaß und Wertungslast können abstrakt-generelle Regeln aufgestellt werden, die so genannten abwägungslenkenden Obersätze. Diese können einer (prozeduralen) Richtigkeitskontrolle zu Grunde gelegt werden, erlauben also eine Falsifikation der Abwägungsentscheidung. Sie sind ihrerseits Gegenstand des wissenschaftlichen Diskurses und tauglicher Inhalt höchstrichterlicher Revisionsentscheidungen und Leitsätze.

5. Maßstab der konkreten Gewichtung und der wertenden Gegenüberstellung im Einzelfall kann mangels der Möglichkeit abstrakt-genereller Regeln hierfür nur die persönliche Überzeugung des zur Entscheidung berufenen Organs sein. Damit ist keine Erweiterung, sondern eine Beschränkung des Anwendungsbereichs richterlicher Eigenwertung intendiert, weil diese auf genau benannte Teile der Abwägungsstruktur eingegrenzt wird, die übrigen Teile aber intersubjektiv auf Einhaltung der abwägungslenkenden Obersätze kontrolliert werden können.

6. Wird die vorstehende Abwägungsstruktur eingehalten, so handelt es sich bei Einzelfallabwägungen um rationale Entscheidungen, weil sie mit Hilfe einer sachlichen Begründung auf die Prämissen - Sachverhalt und Normtext - zurückführbar sind. Damit genügen sie zugleich den rechtsstaatlichen Anforderungen an die Entscheidungsfindung. 